Supporting Information

\title{
Thiocyanation and 2-amino-1,3-thiazole formation in water using recoverable and reusable glycosylated resorcin[4]arene cavitands
}

\author{
Ali A. Husain and Prof. Dr. Kirpal S. Bisht* \\ Department of Chemistry, University of South Florida, 4202 East Fowler Avenue, Tampa, \\ Florida, 33620, USA. \\ E-mail:kbisht@usf.edu
}

\section{Table of Contents}

NMR Spectra

Mass Spectra .523

\section{General}

${ }^{1} \mathrm{H}$ - and ${ }^{13} \mathrm{C}-\mathrm{NMR}$ spectra were recorded on a Bruker DRX-250 and Inova 400 spectrometers. Sample concentrations were about $10 \%(\mathrm{w} / \mathrm{v})$ in $\mathrm{CDCl}_{3}$ and DMSO- $\mathrm{d}_{6}$ and the $J$ values are given in $\mathrm{Hz}$. The mass spectral analyses were performed on an Aligent Technologies 6540 UHD Accurate-Mass Q-TOF LC/MS. 


\section{NMR Spectra}

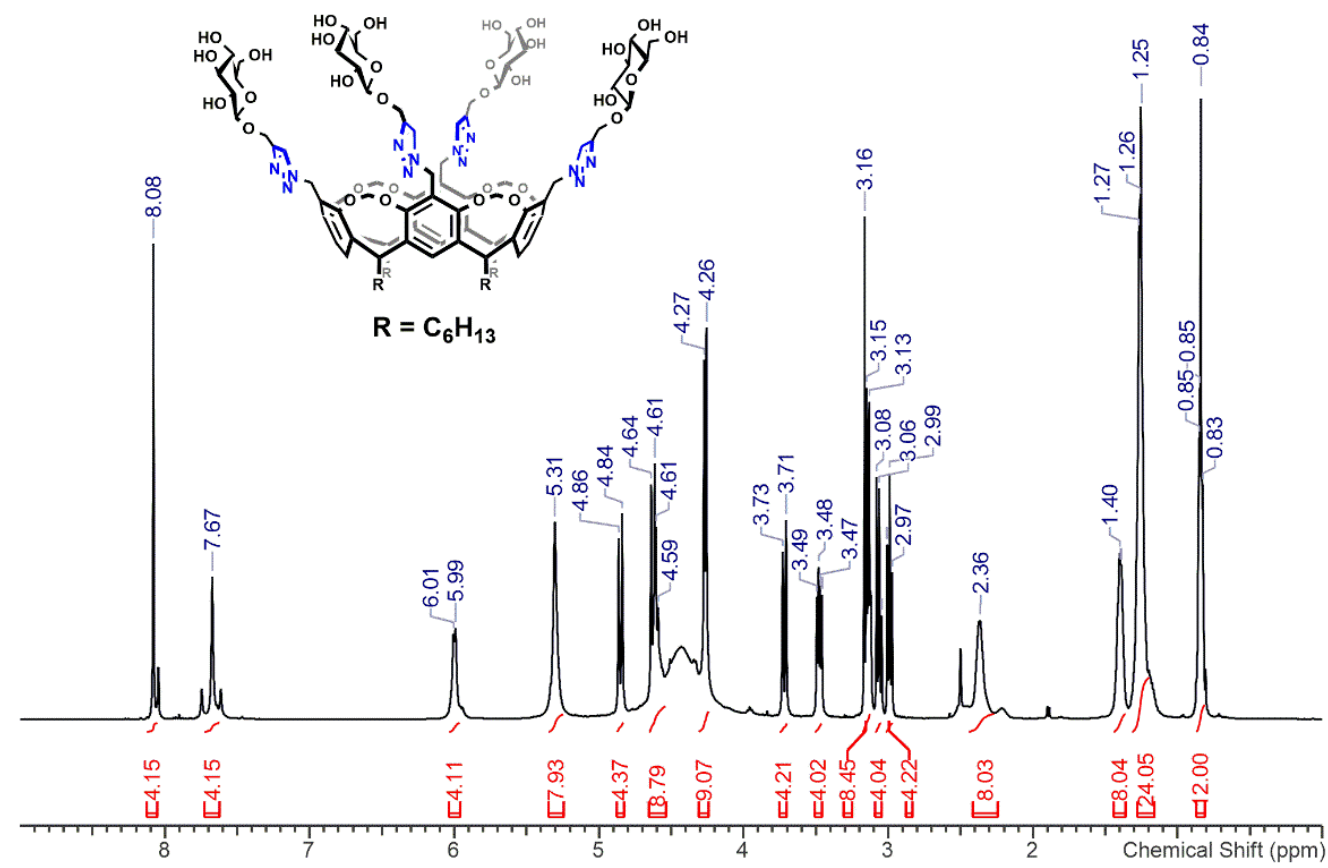

Figure S1. ${ }^{1} \mathrm{H}-\mathrm{NMR}$ spectrum of RCG1 (DMSO-d 6 , $400 \mathrm{MHz}, 25^{\circ} \mathrm{C}$ ).
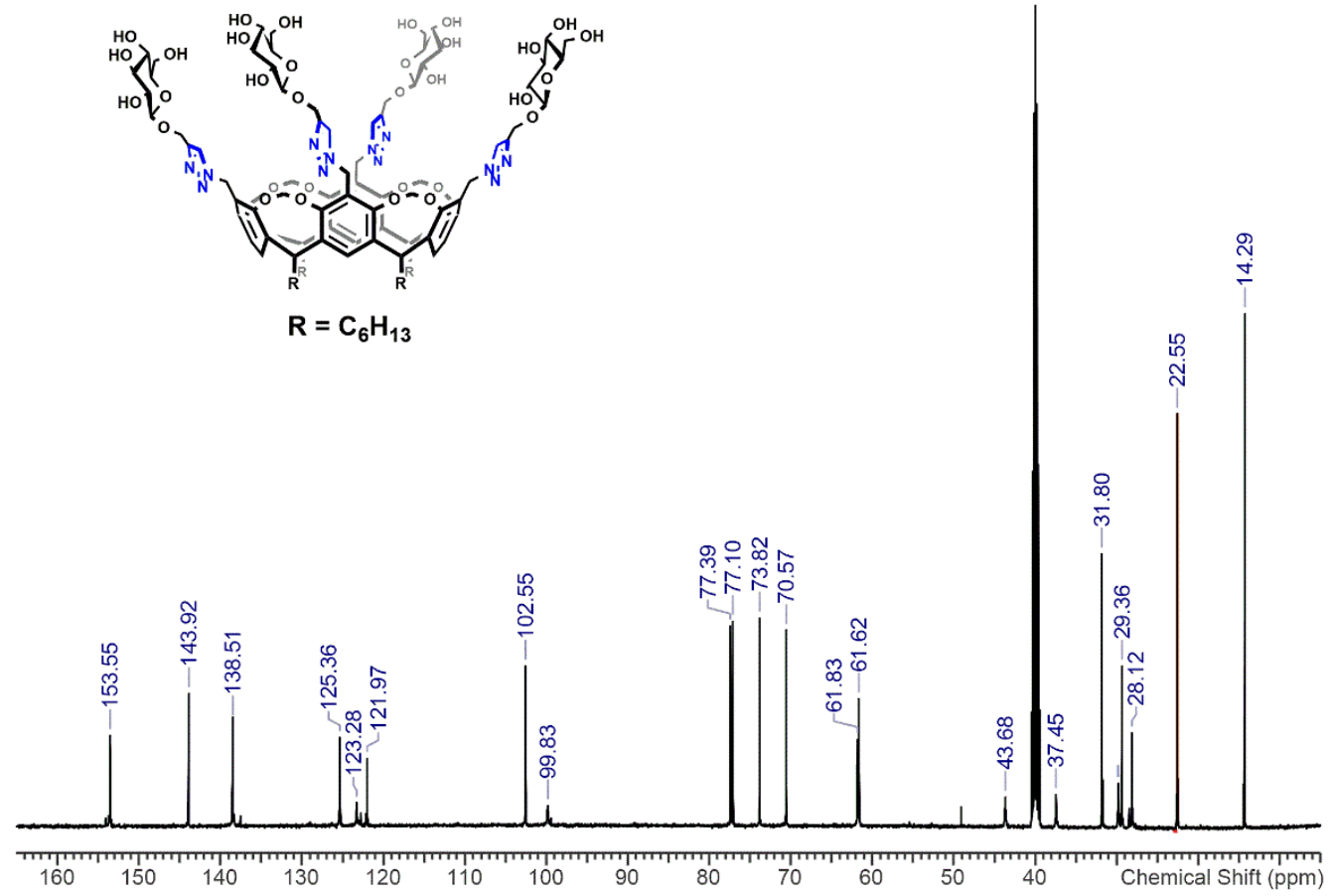

Figure S2. ${ }^{13} \mathrm{C}\{\mathrm{H}\}$-NMR spectrum of RCG1 (DMSO-d 6 , $100 \mathrm{MHz}, 25{ }^{\circ} \mathrm{C}$ ). 


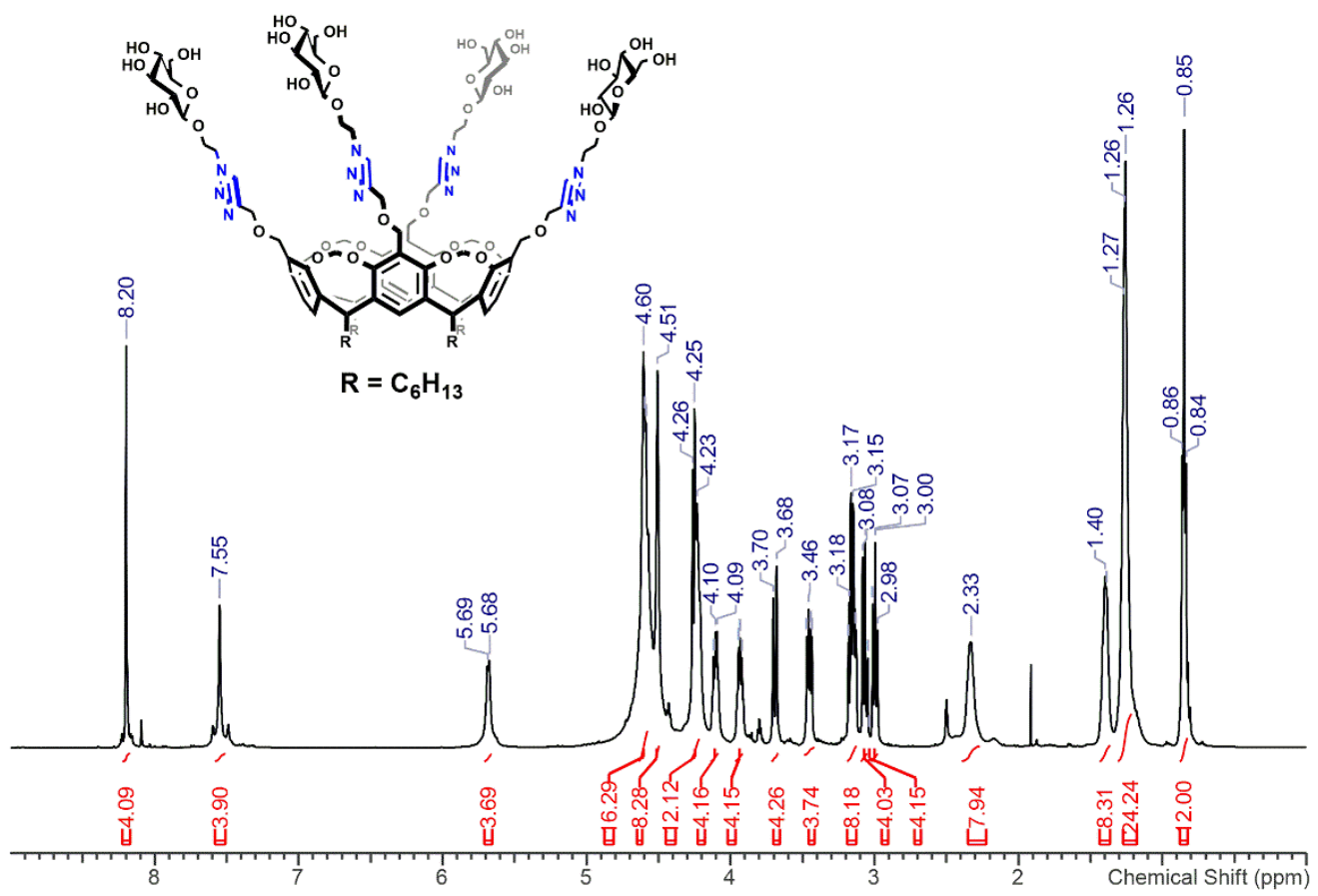

Figure S3. ${ }^{1} \mathrm{H}-\mathrm{NMR}$ spectrum of RCG3 (DMSO-d $6,400 \mathrm{MHz}, 25^{\circ} \mathrm{C}$ ).
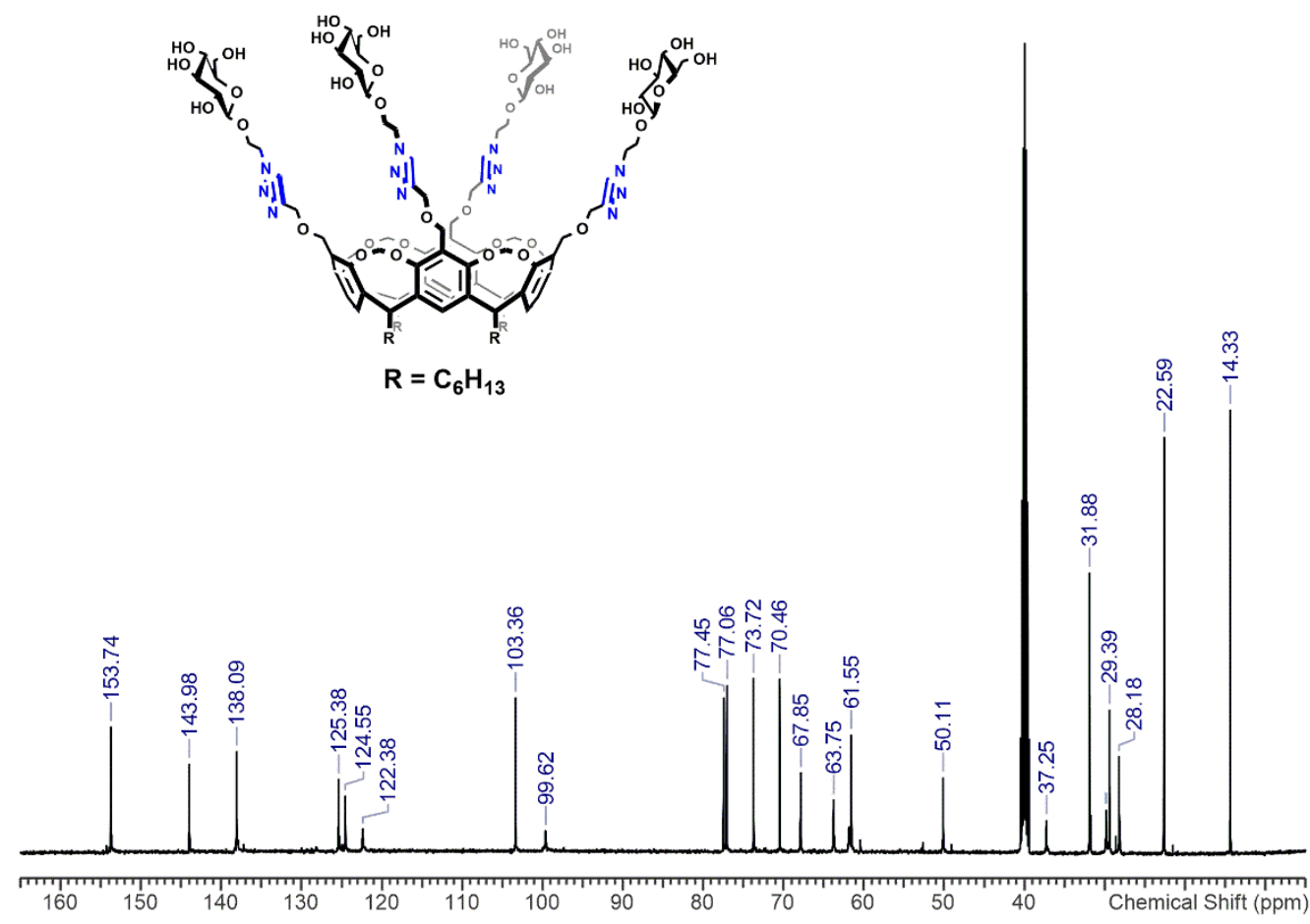

Figure S4. ${ }^{13} \mathrm{C}\{\mathrm{H}\}$-NMR spectrum of RCG3 $\left(\right.$ DMSO-d $_{6}, 100 \mathrm{MHz}, 2{ }^{\circ} \mathrm{C}$ ). 


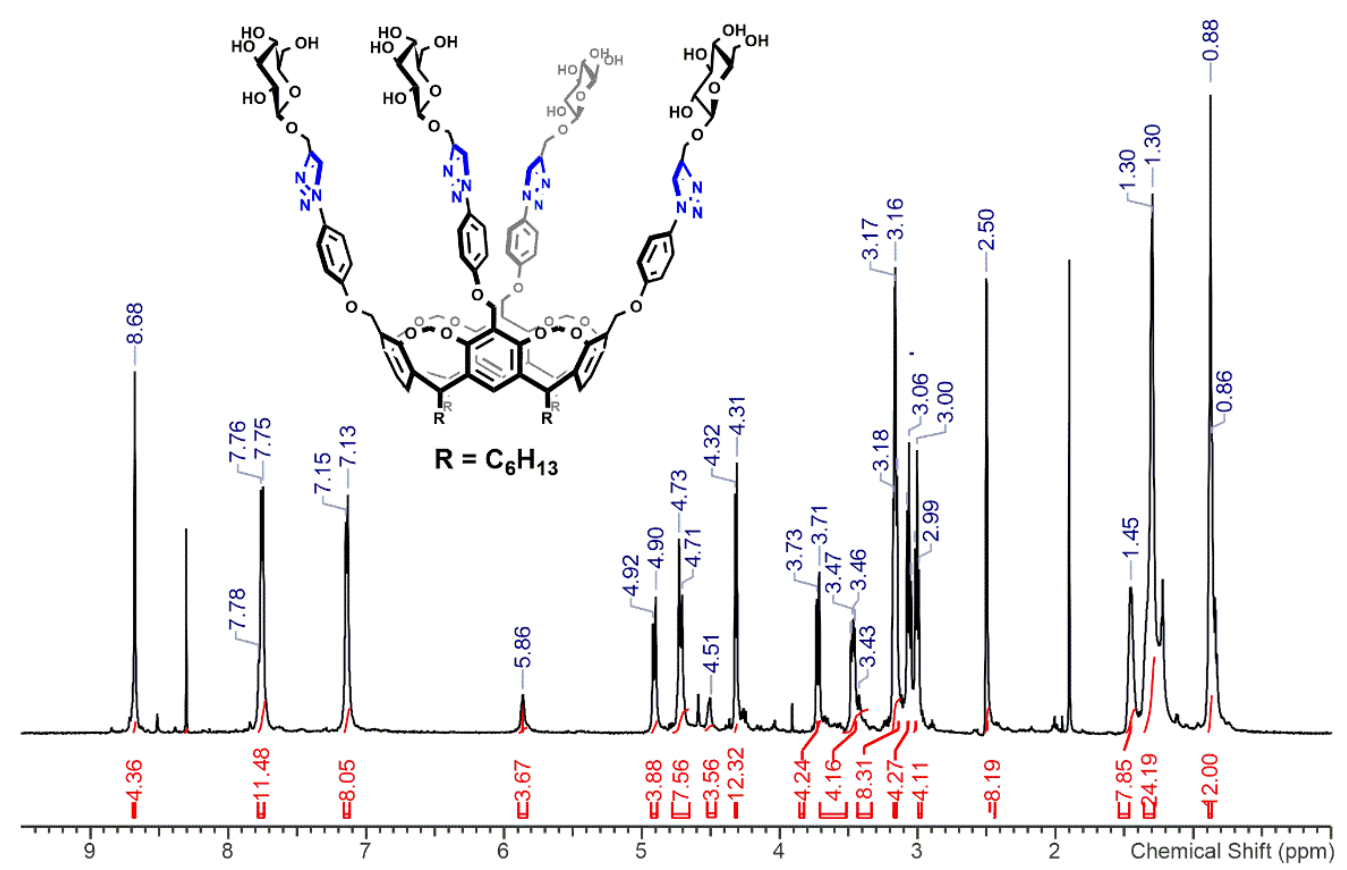

Figure S5. ${ }^{1} \mathrm{H}-\mathrm{NMR}$ spectrum of RCG5 (DMSO-d 6 , $400 \mathrm{MHz}, 25{ }^{0} \mathrm{C}$ ).

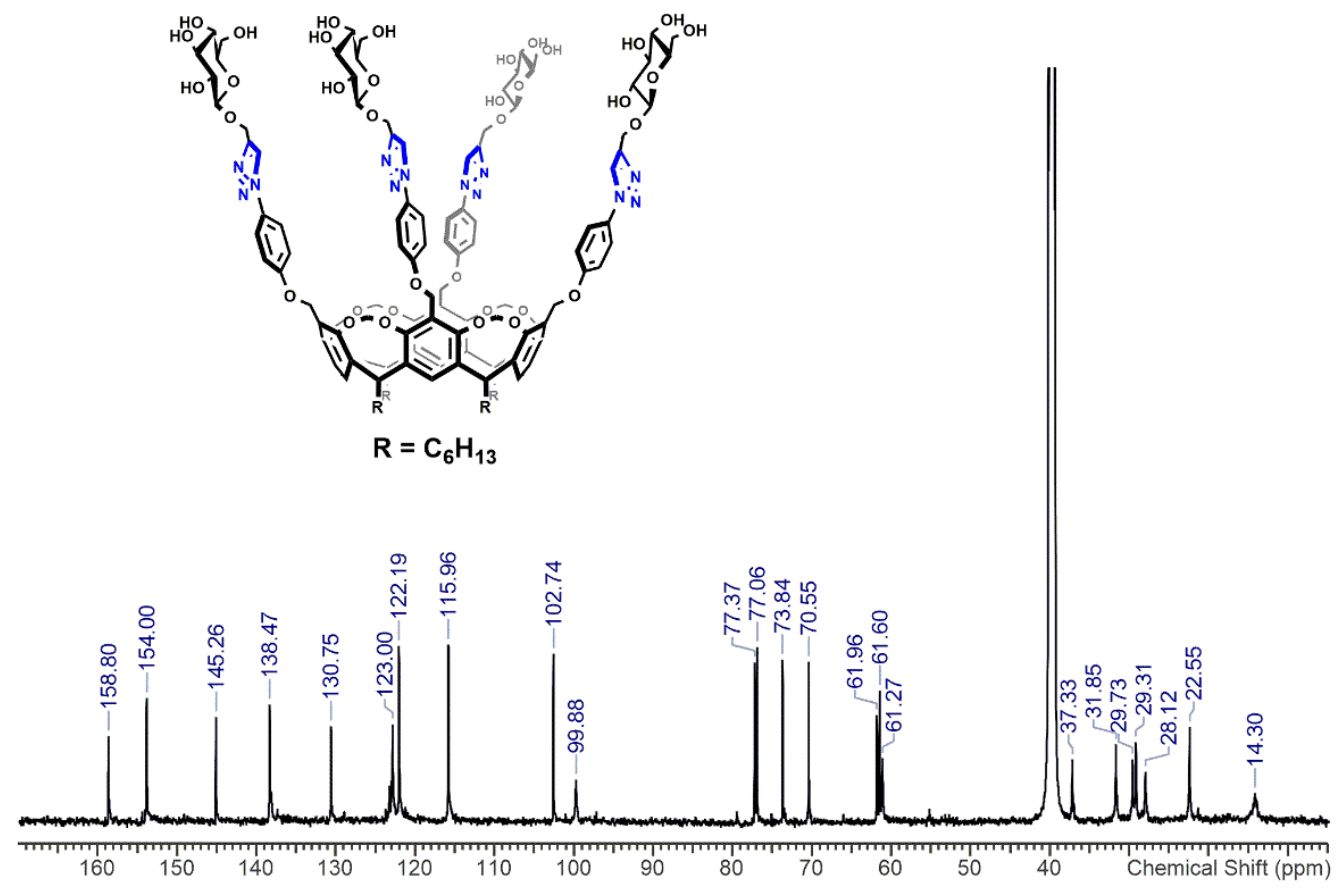

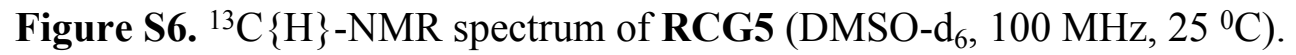




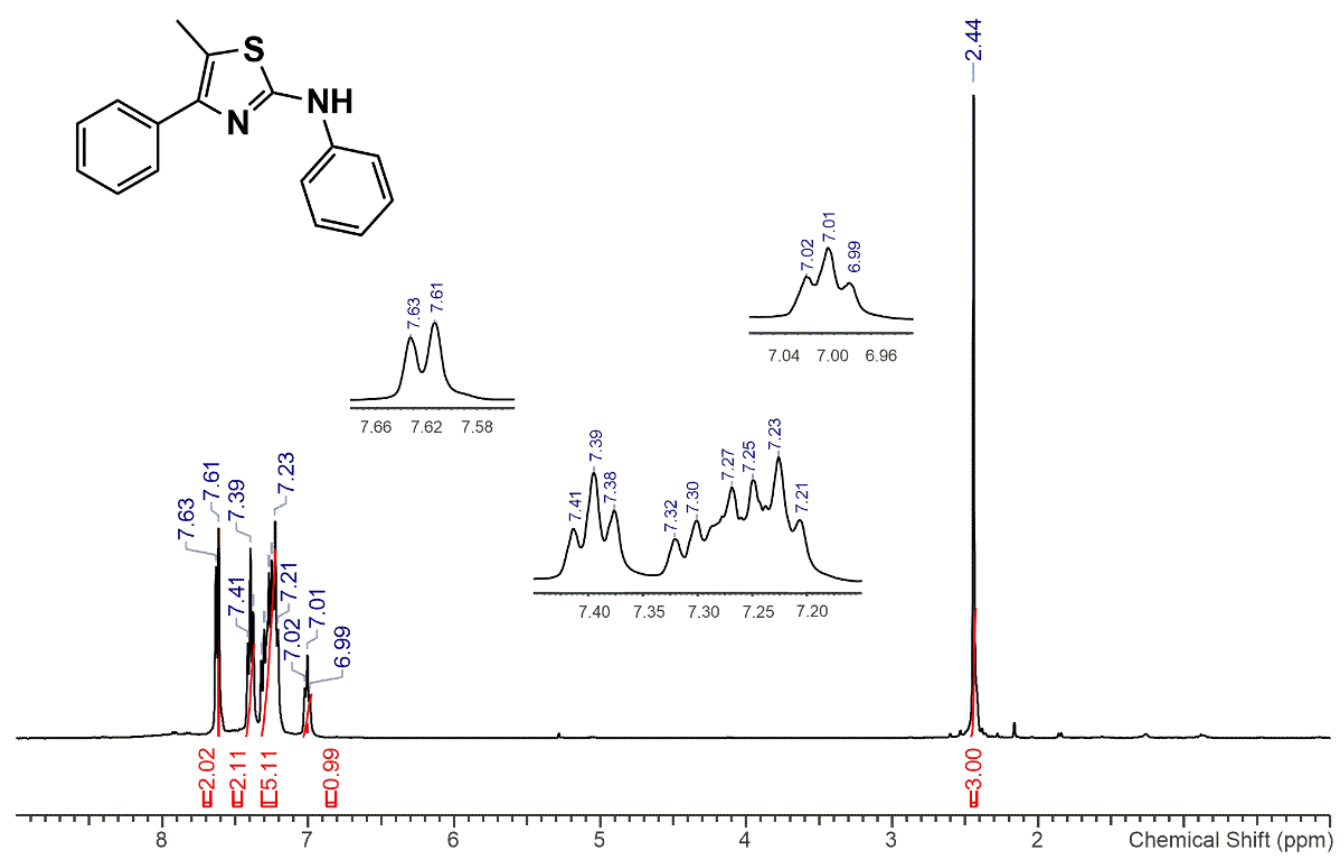

Figure S7. ${ }^{1} \mathrm{H}-\mathrm{NMR}$ spectrum of thiazole $\mathbf{1 b}\left(\mathrm{CDCl}_{3}, 400 \mathrm{MHz}, 25{ }^{\circ} \mathrm{C}\right)$.

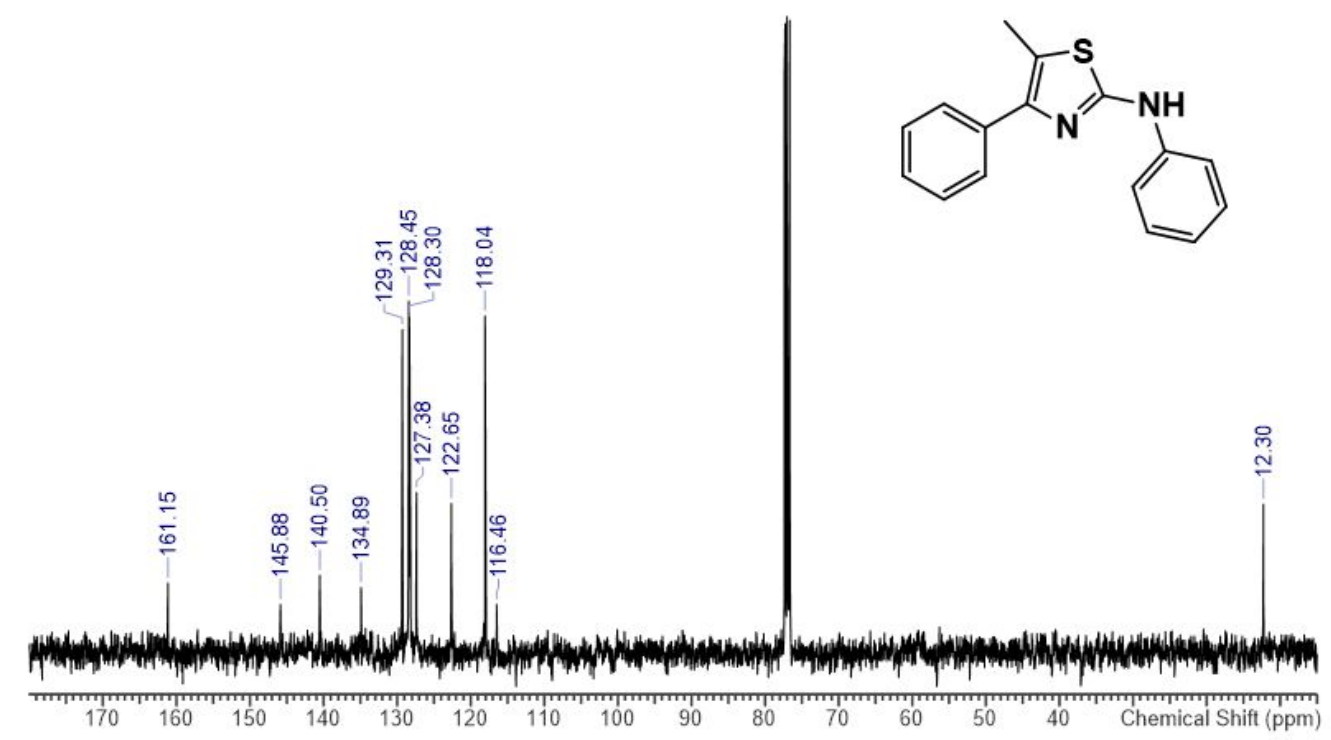

Figure S8. ${ }^{13} \mathrm{C}\{\mathrm{H}\}$-NMR spectrum of thiazole $\mathbf{1 b}\left(\mathrm{CDCl}_{3}, 100 \mathrm{MHz}, 25{ }^{\circ} \mathrm{C}\right)$. 
<smiles>Cc1sc(Nc2ccccc2O)nc1-c1ccccc1</smiles>
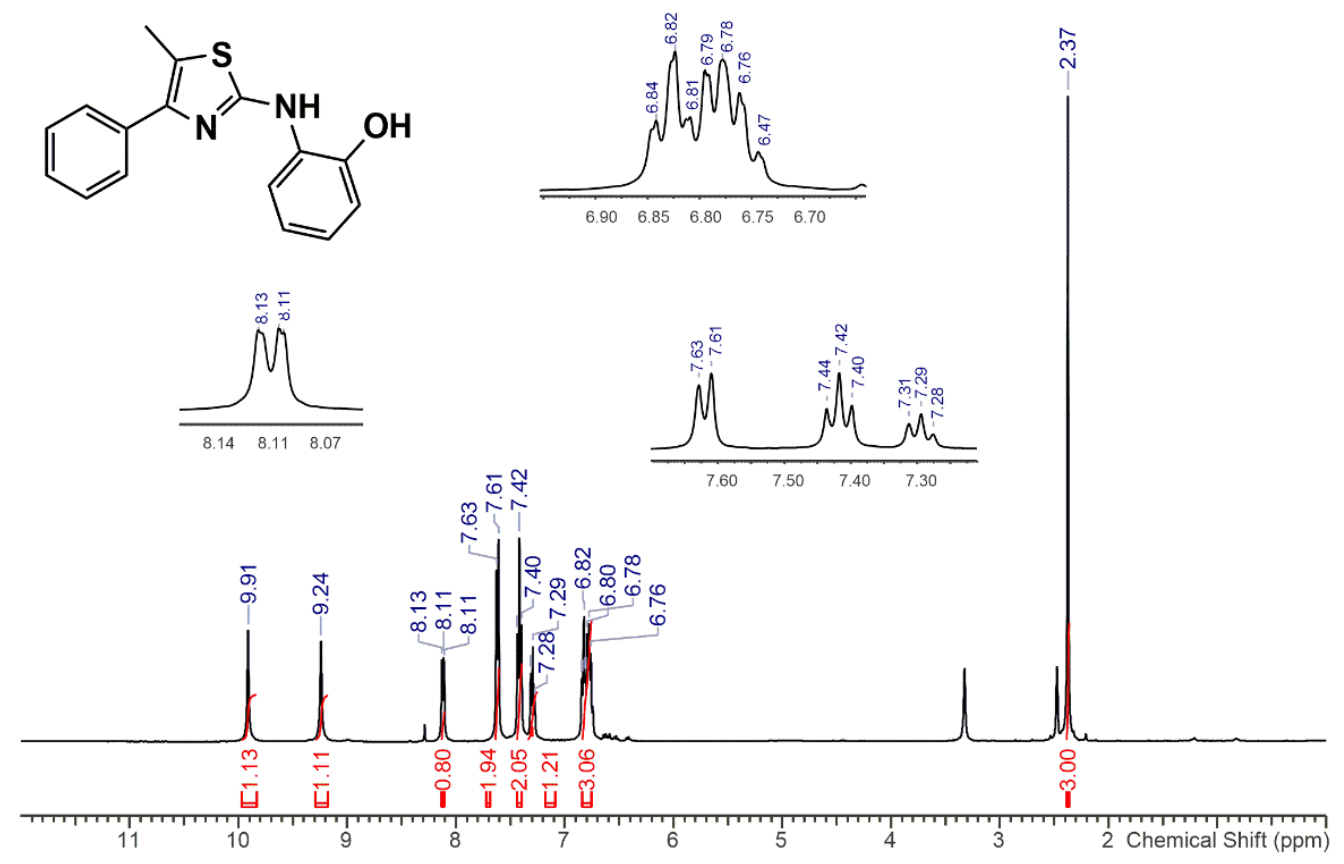

Figure S9. ${ }^{1} \mathrm{H}-\mathrm{NMR}$ spectrum of thiazole $\mathbf{2 b}\left(\mathrm{DMSO}_{6}, 400 \mathrm{MHz}, 25{ }^{\circ} \mathrm{C}\right)$.<smiles>Cc1sc(Nc2ccccc2O)nc1-c1ccccc1</smiles>

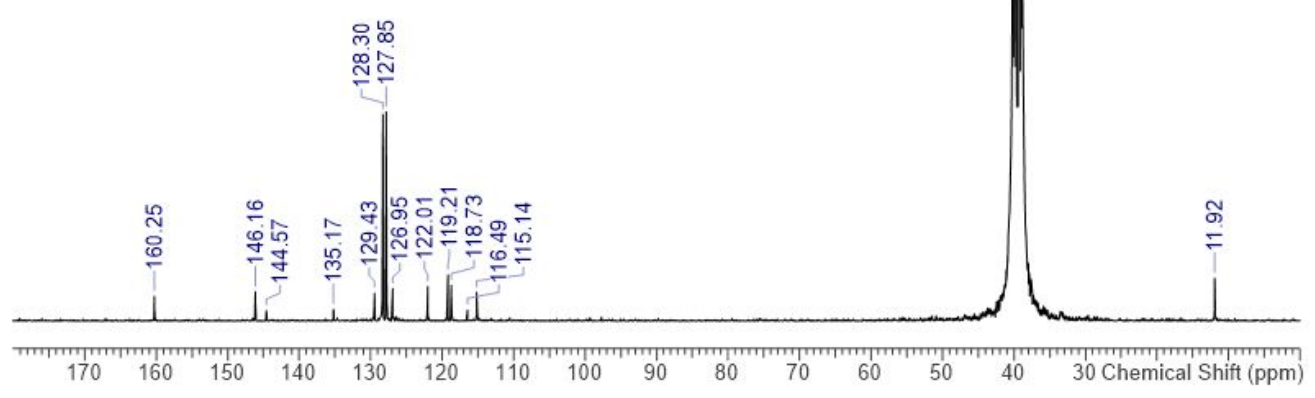

Figure S10. ${ }^{13} \mathrm{C}\{\mathrm{H}\}-\mathrm{NMR}$ spectrum of thiazole $\mathbf{2 b}\left(\right.$ DMSO-d $\left._{6}, 100 \mathrm{MHz}, 2{ }^{\circ} \mathrm{C}\right)$. 


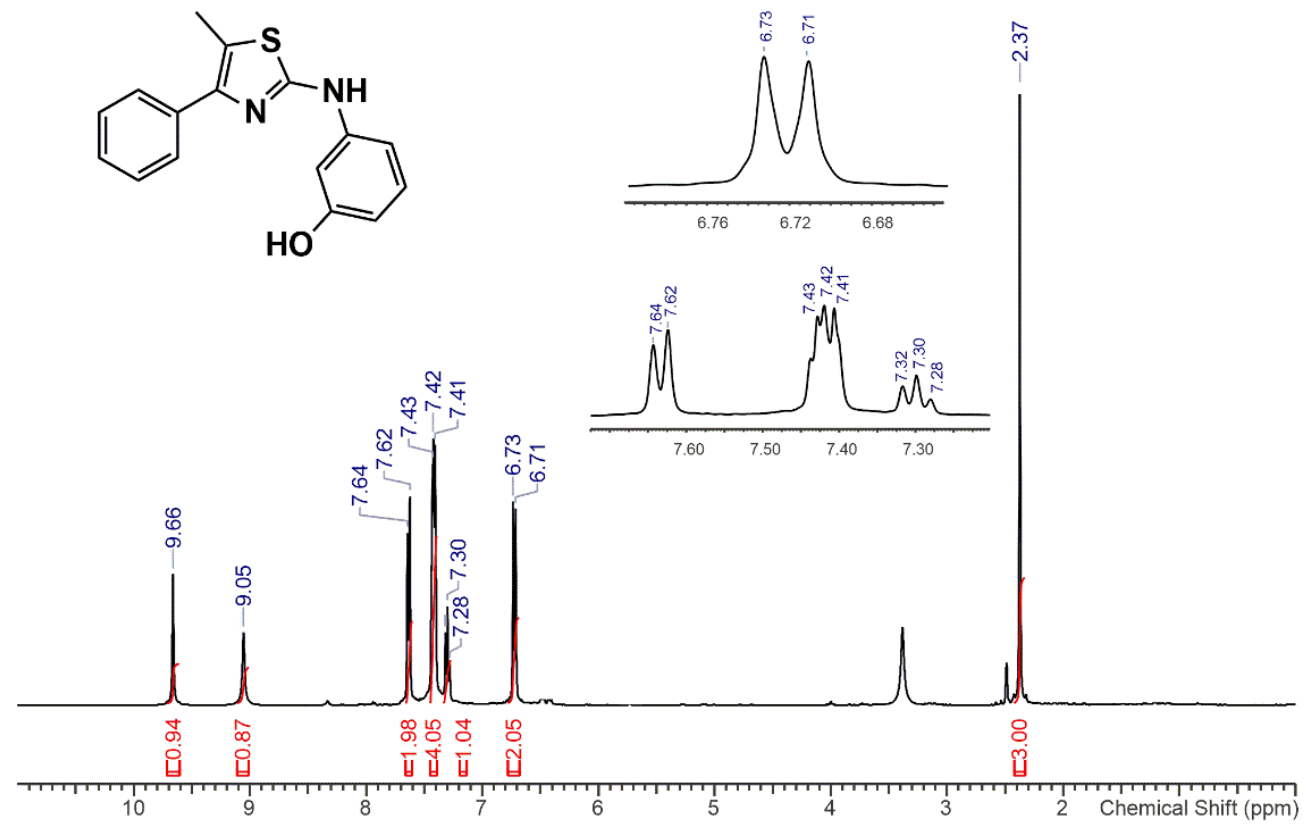

Figure S11. ${ }^{1} \mathrm{H}-\mathrm{NMR}$ spectrum of thiazole $\mathbf{3 b}\left(\mathrm{DMSO}_{6} \mathrm{~d}_{6}, 400 \mathrm{MHz}, 25{ }^{0} \mathrm{C}\right)$.

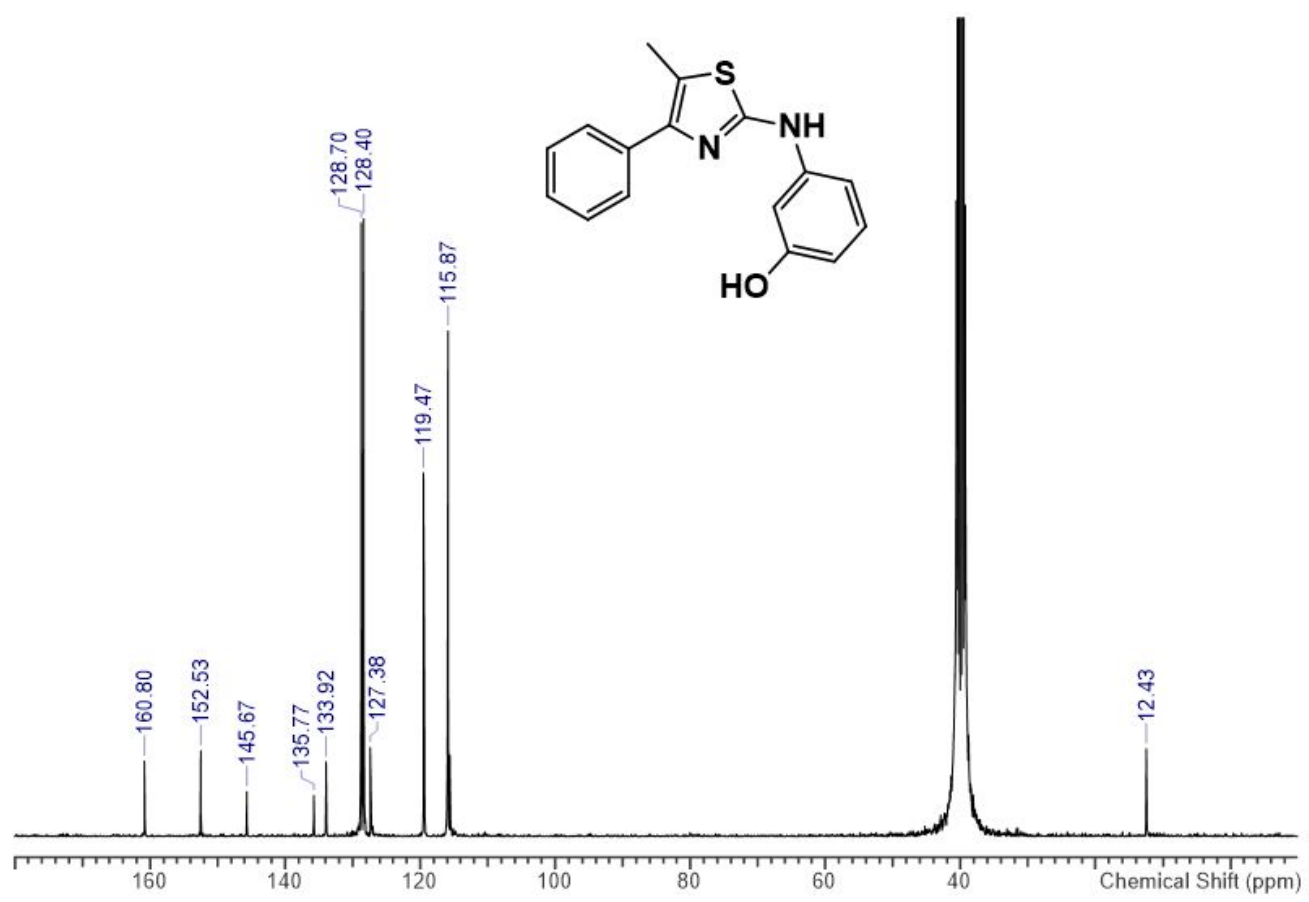

Figure S12. ${ }^{13} \mathrm{C}\{\mathrm{H}\}-\mathrm{NMR}$ spectrum of thiazole $3 \mathbf{b}\left(\mathrm{DMSO}-\mathrm{d}_{6}, 100 \mathrm{MHz}, 25{ }^{0} \mathrm{C}\right)$. 


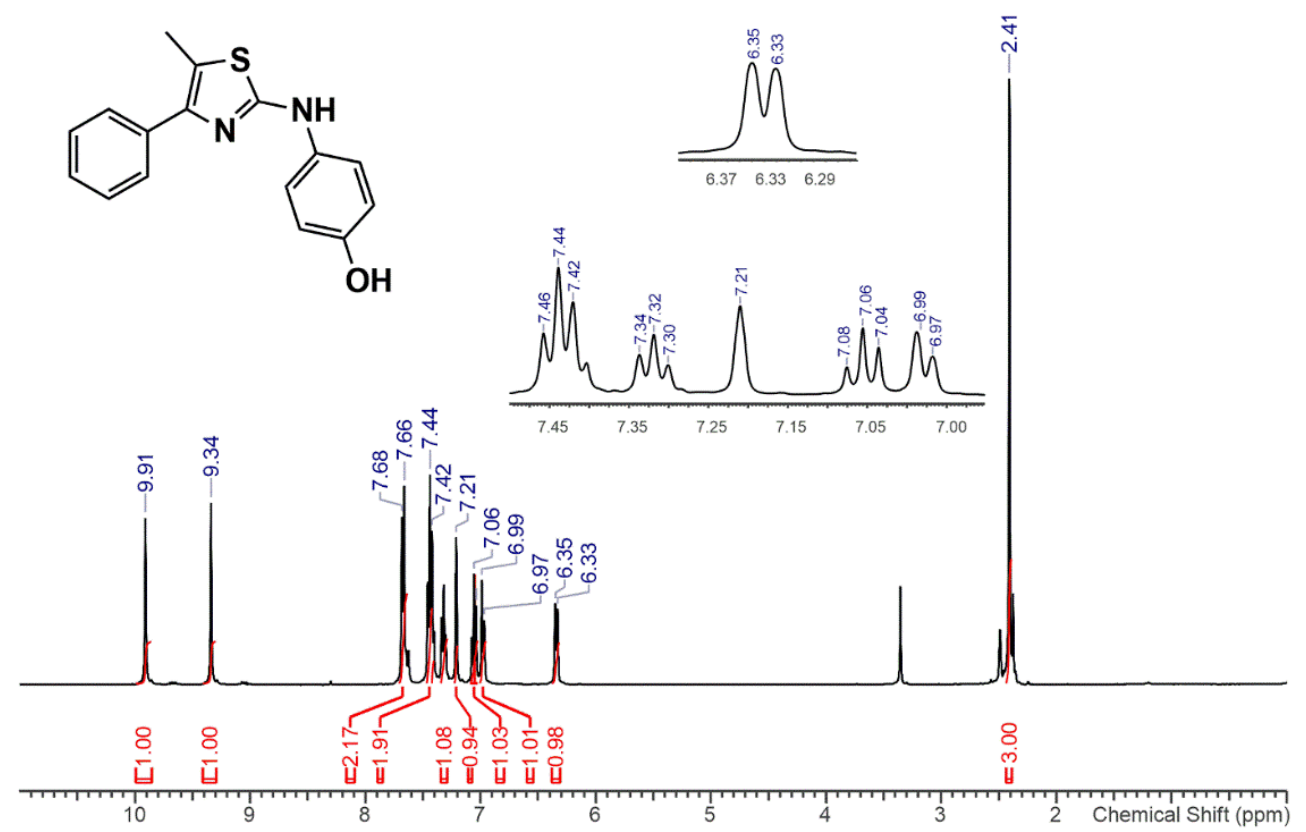

Figure S13. ${ }^{1} \mathrm{H}-\mathrm{NMR}$ spectrum of thiazole $4 \mathbf{b}\left(\mathrm{DMSO}_{6}, 400 \mathrm{MHz}, 25{ }^{\circ} \mathrm{C}\right)$.

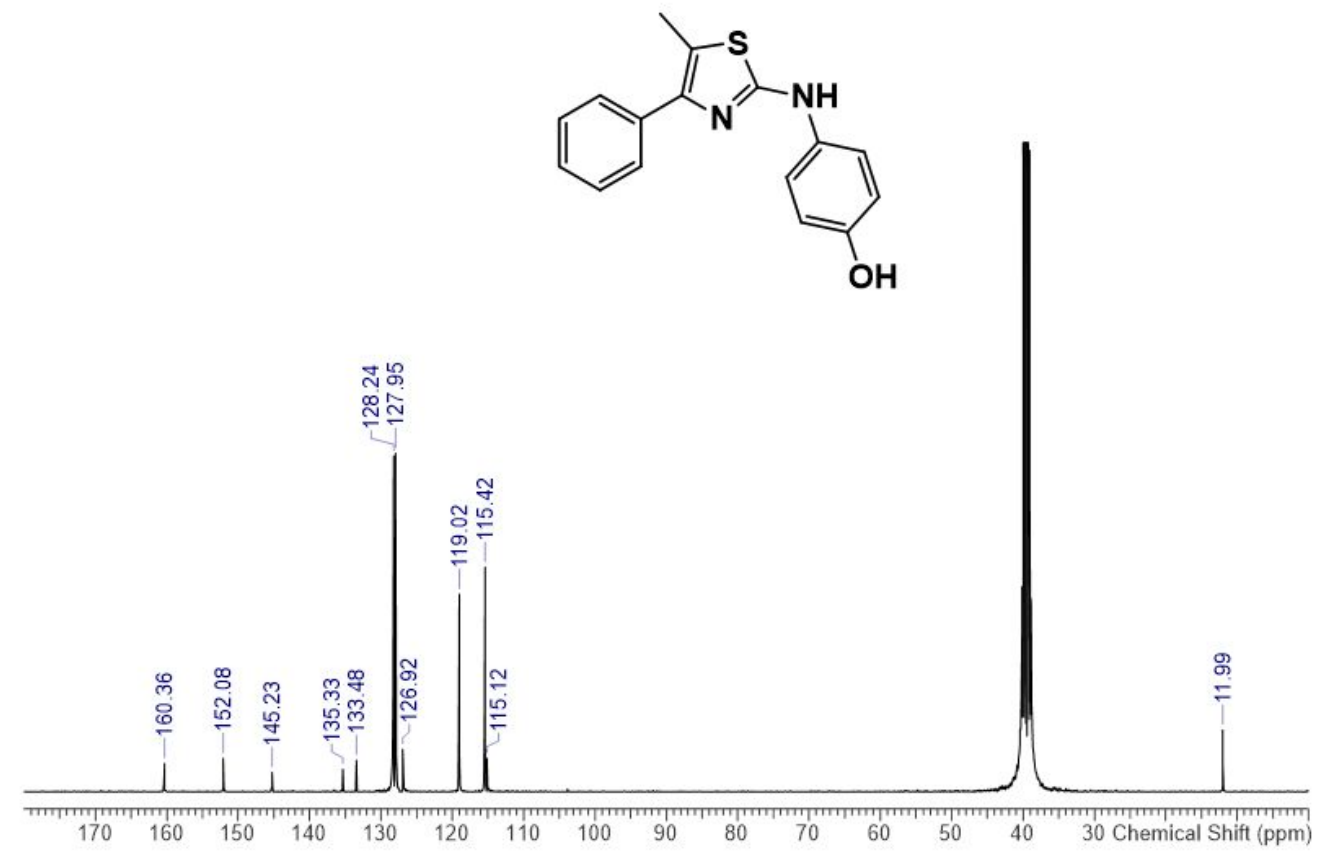

Figure S14. ${ }^{13} \mathrm{C}\{\mathrm{H}\}-\mathrm{NMR}$ spectrum of thiazole $4 \mathbf{b}\left(\mathrm{DMSO}-\mathrm{d}_{6}, 100 \mathrm{MHz}, 25{ }^{\circ} \mathrm{C}\right)$. 


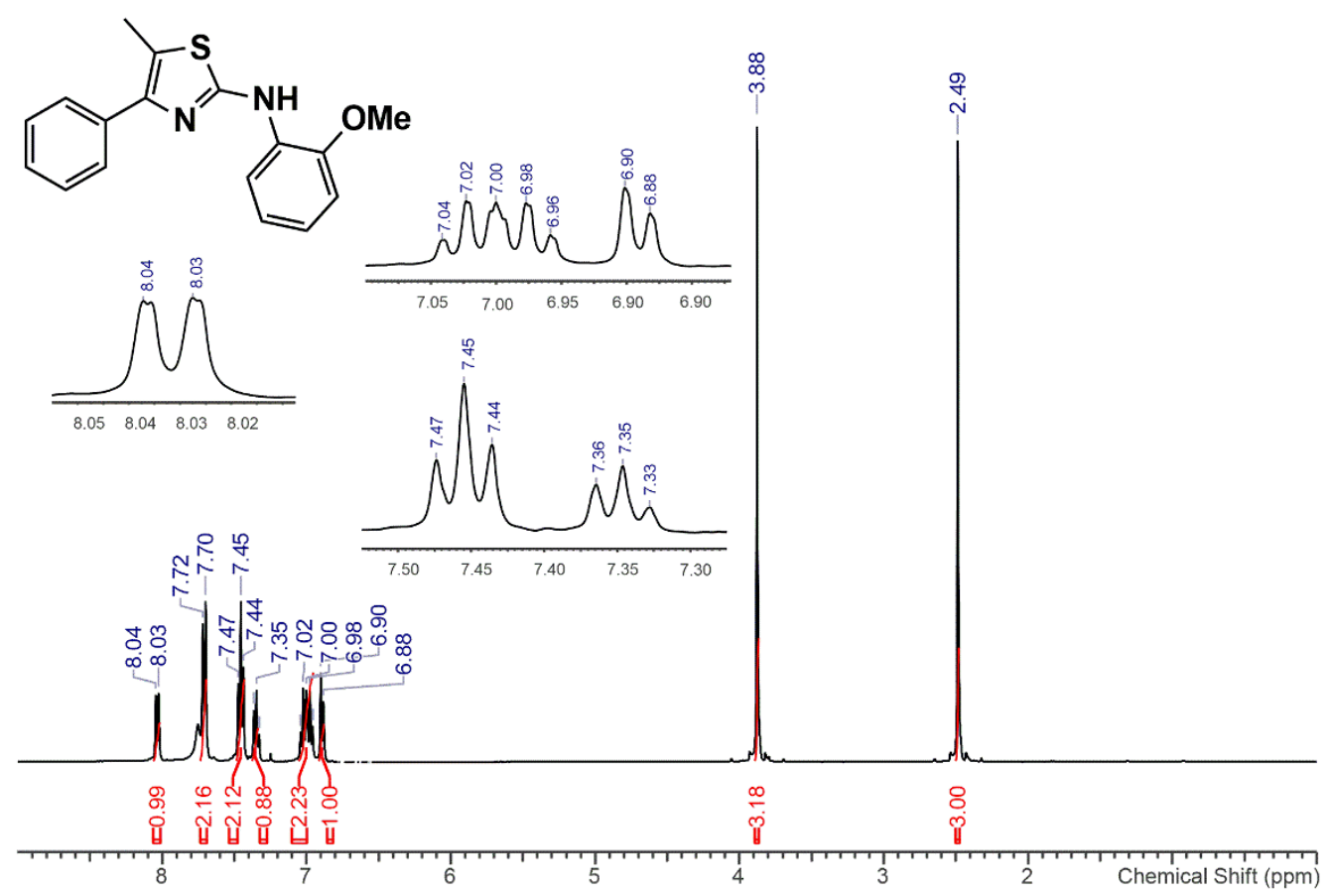

Figure S15. ${ }^{1} \mathrm{H}-\mathrm{NMR}$ spectrum of thiazole $\mathbf{5 b}\left(\mathrm{CDCl}_{3}, 400 \mathrm{MHz}, 25^{\circ} \mathrm{C}\right)$.

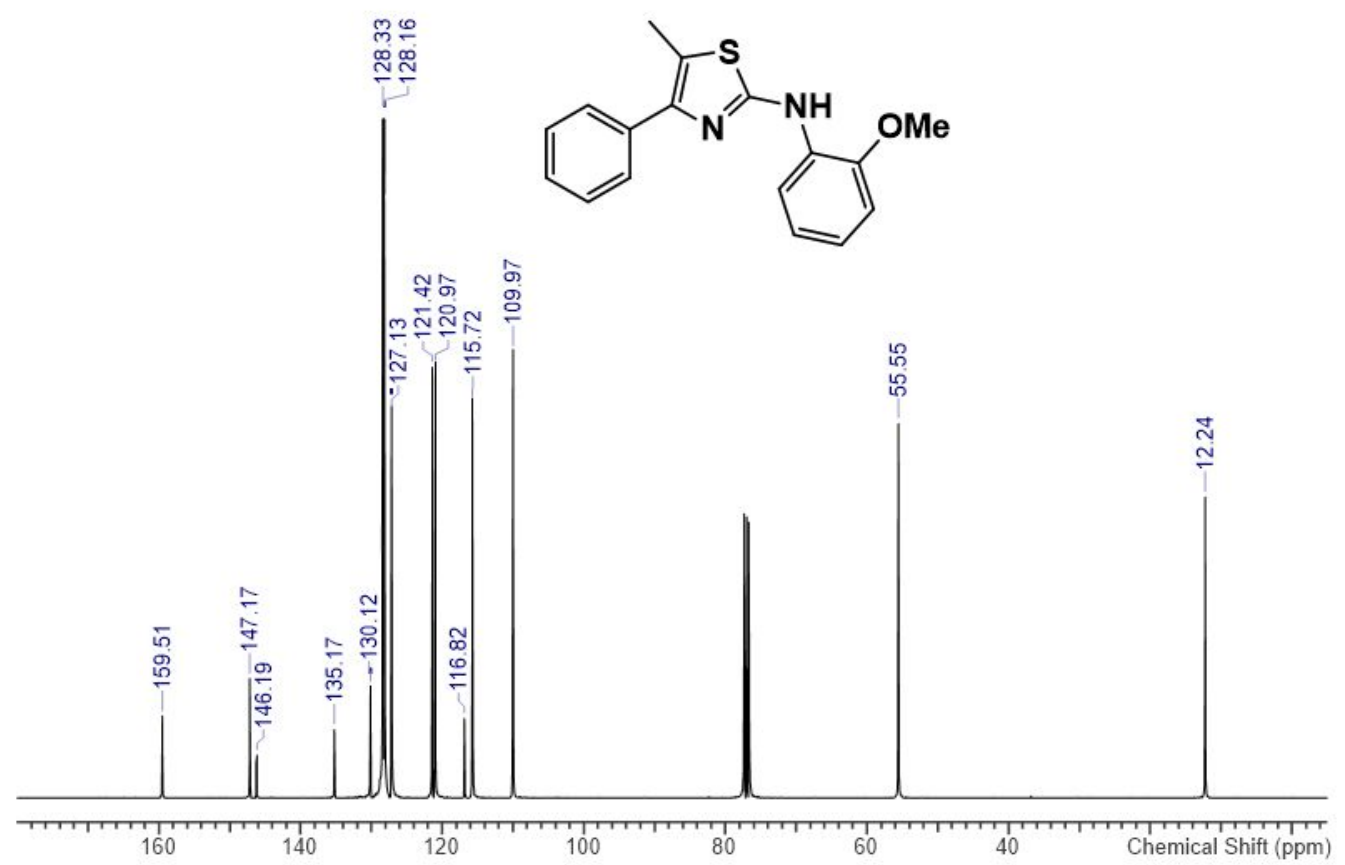

Figure S16. ${ }^{13} \mathrm{C}\{\mathrm{H}\}$-NMR spectrum of thiazole $\mathbf{5 b}\left(\mathrm{CDCl}_{3}, 100 \mathrm{MHz}, 25{ }^{\circ} \mathrm{C}\right)$. 


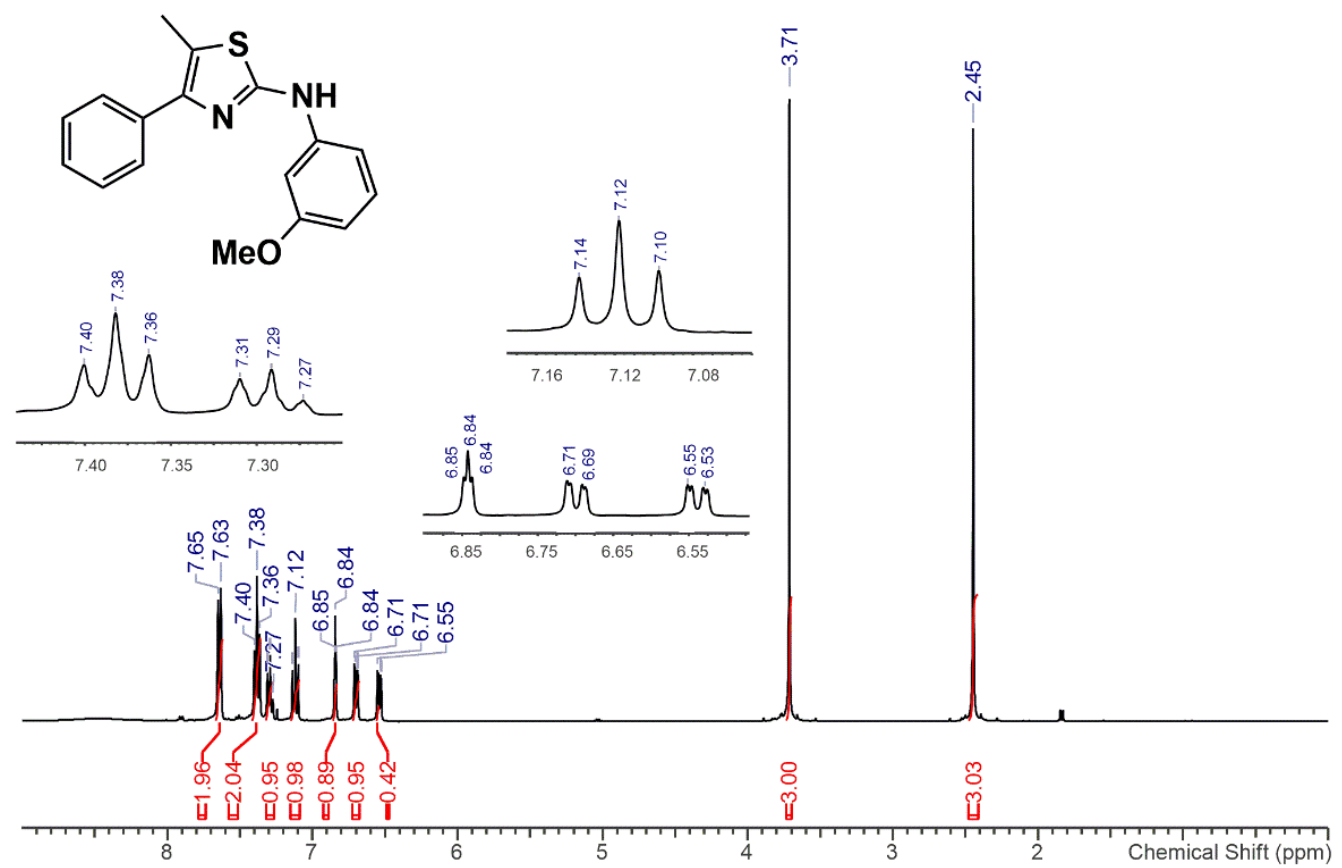

Figure S17. ${ }^{1} \mathrm{H}-\mathrm{NMR}$ spectrum of thiazole $\mathbf{6 b}\left(\mathrm{CDCl}_{3}, 400 \mathrm{MHz}, 25{ }^{0} \mathrm{C}\right)$.

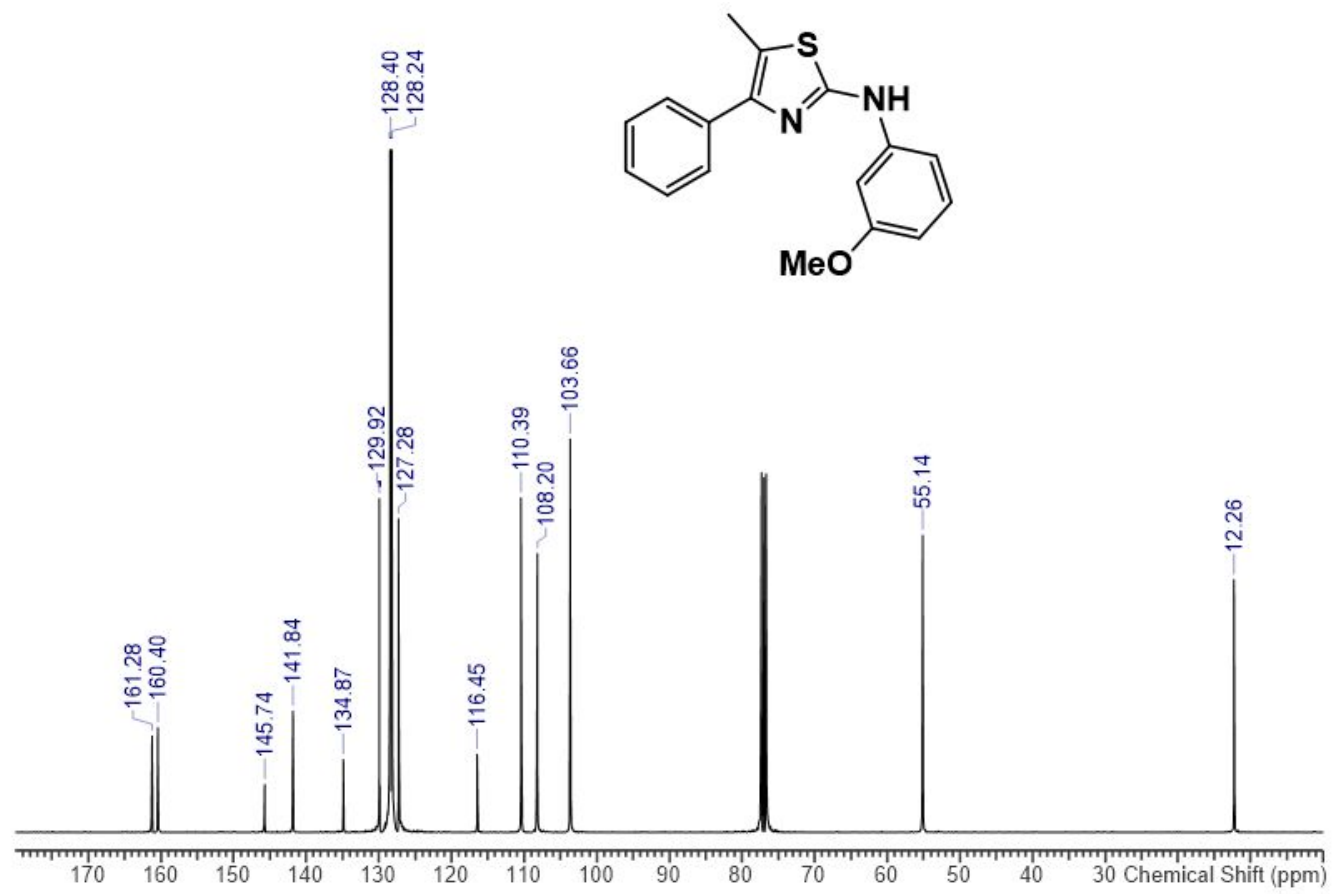

Figure S18. ${ }^{13} \mathrm{C}\{\mathrm{H}\}-\mathrm{NMR}$ spectrum of thiazole $\mathbf{6 b}\left(\mathrm{CDCl}_{3}, 100 \mathrm{MHz}, 25{ }^{0} \mathrm{C}\right)$. 


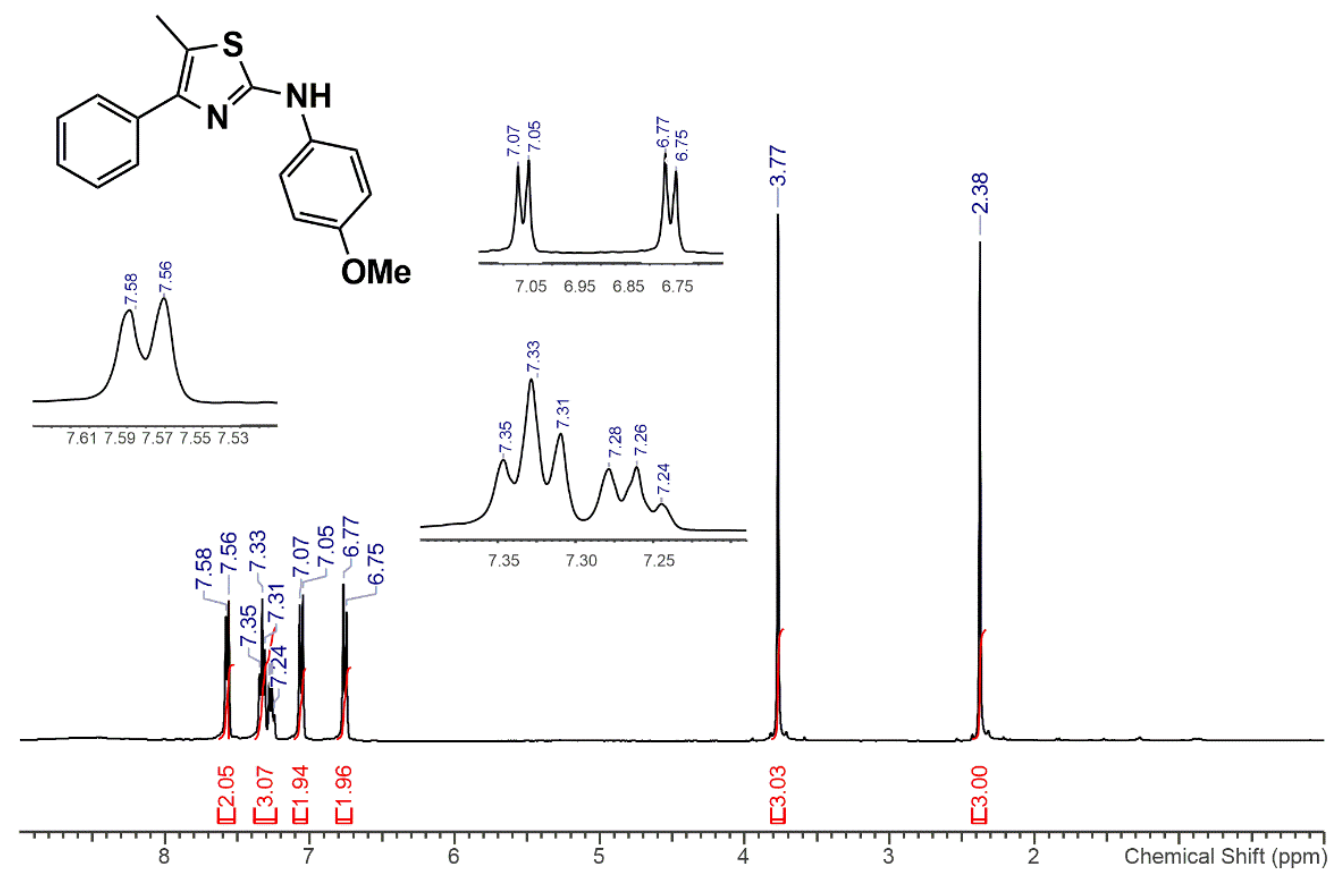

Figure S19. ${ }^{1} \mathrm{H}-\mathrm{NMR}$ spectrum of thiazole $7 \mathbf{b}\left(\mathrm{CDCl}_{3}, 400 \mathrm{MHz}, 25{ }^{\circ} \mathrm{C}\right)$.

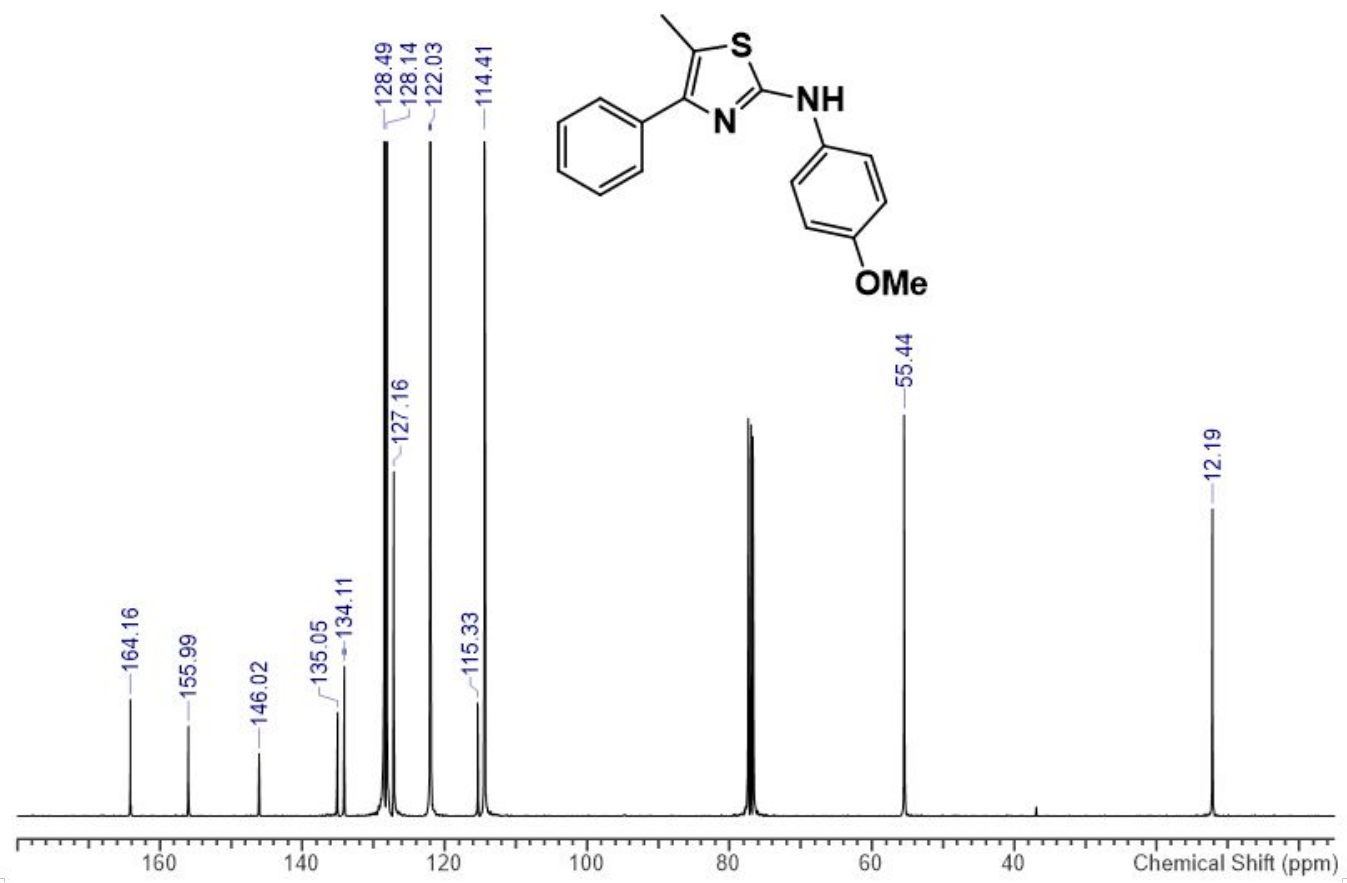

Figure S20. ${ }^{13} \mathrm{C}\{\mathrm{H}\}$-NMR spectrum of thiazole $7 \mathbf{b}\left(\mathrm{CDCl}_{3}, 100 \mathrm{MHz}, 25{ }^{\circ} \mathrm{C}\right)$. 


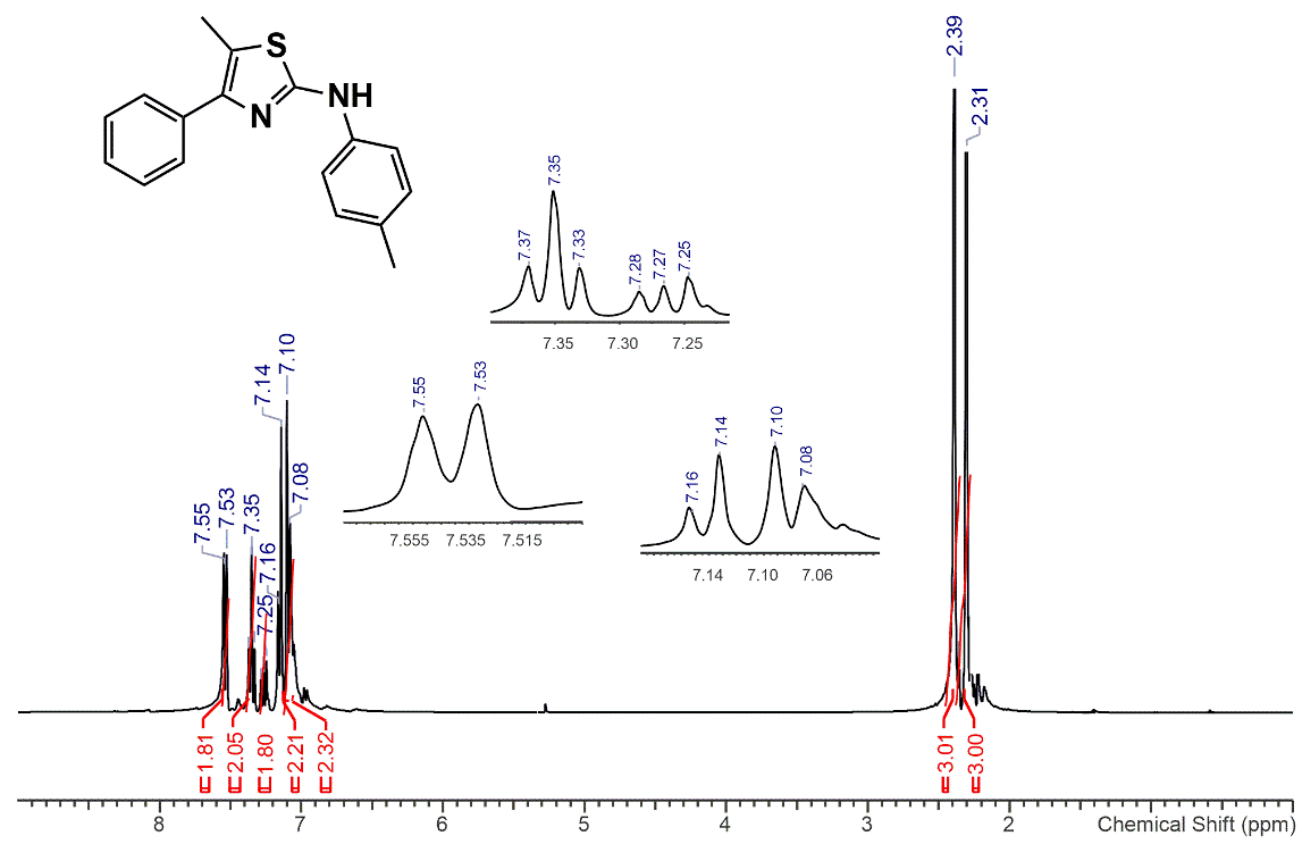

Figure S21. ${ }^{1} \mathrm{H}-\mathrm{NMR}$ spectrum of thiazole $\mathbf{8 b}\left(\mathrm{CDCl}_{3}, 400 \mathrm{MHz}, 25{ }^{0} \mathrm{C}\right)$.

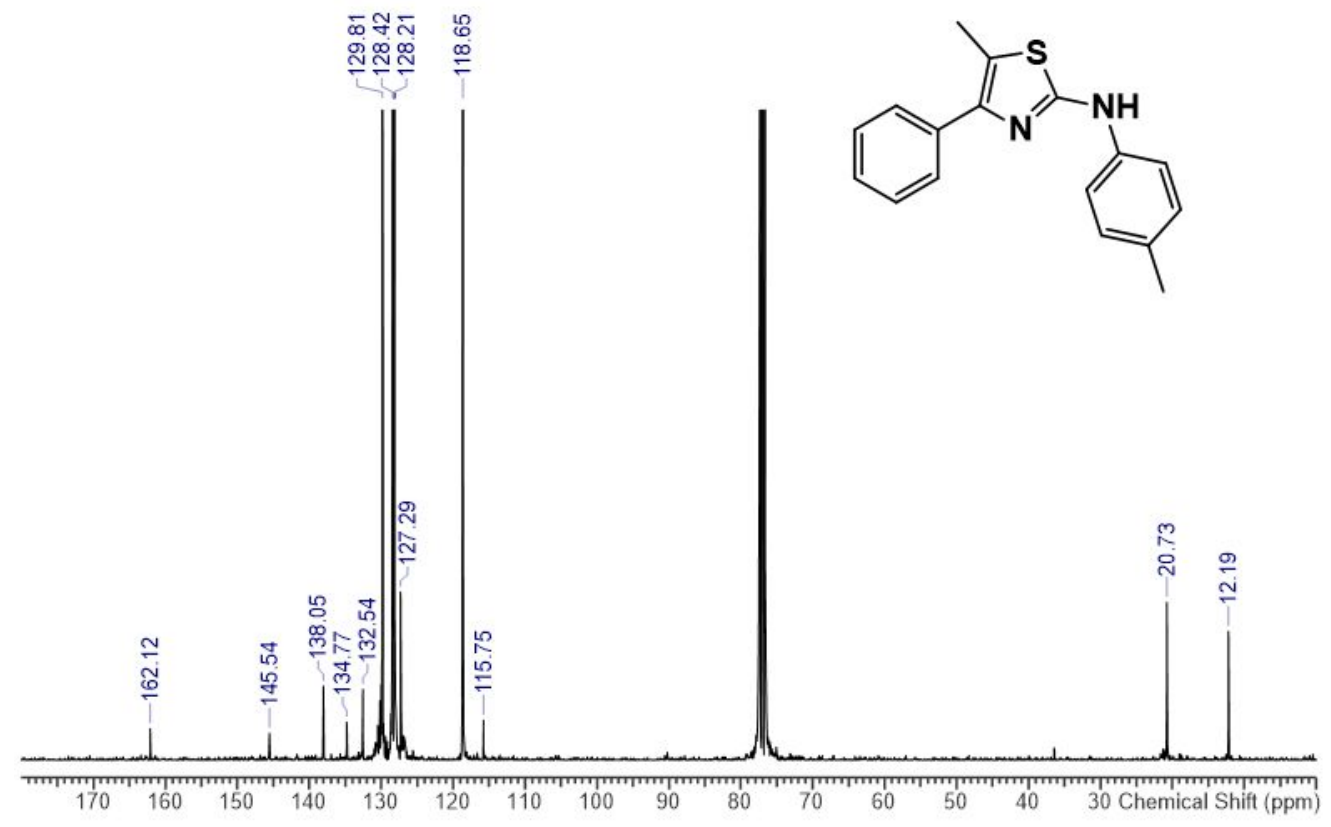

Figure S22. ${ }^{13} \mathrm{C}\{\mathrm{H}\}$-NMR spectrum of thiazole $\mathbf{8 b}\left(\mathrm{CDCl}_{3}, 100 \mathrm{MHz}, 25{ }^{0} \mathrm{C}\right)$. 


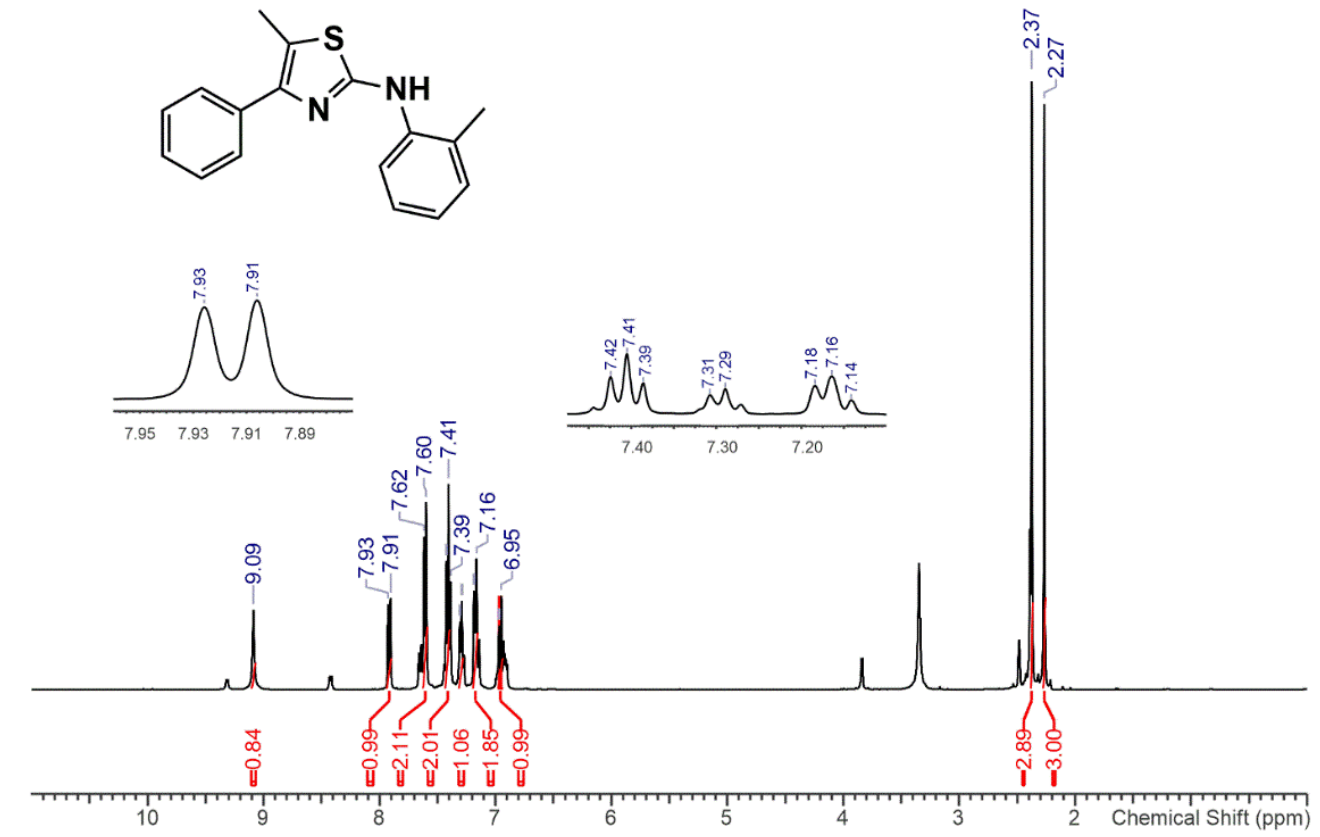

Figure S23. ${ }^{1} \mathrm{H}-\mathrm{NMR}$ spectrum of thiazole 9b (DMSO-d 6 , $\left.400 \mathrm{MHz}, 25{ }^{0} \mathrm{C}\right)$.

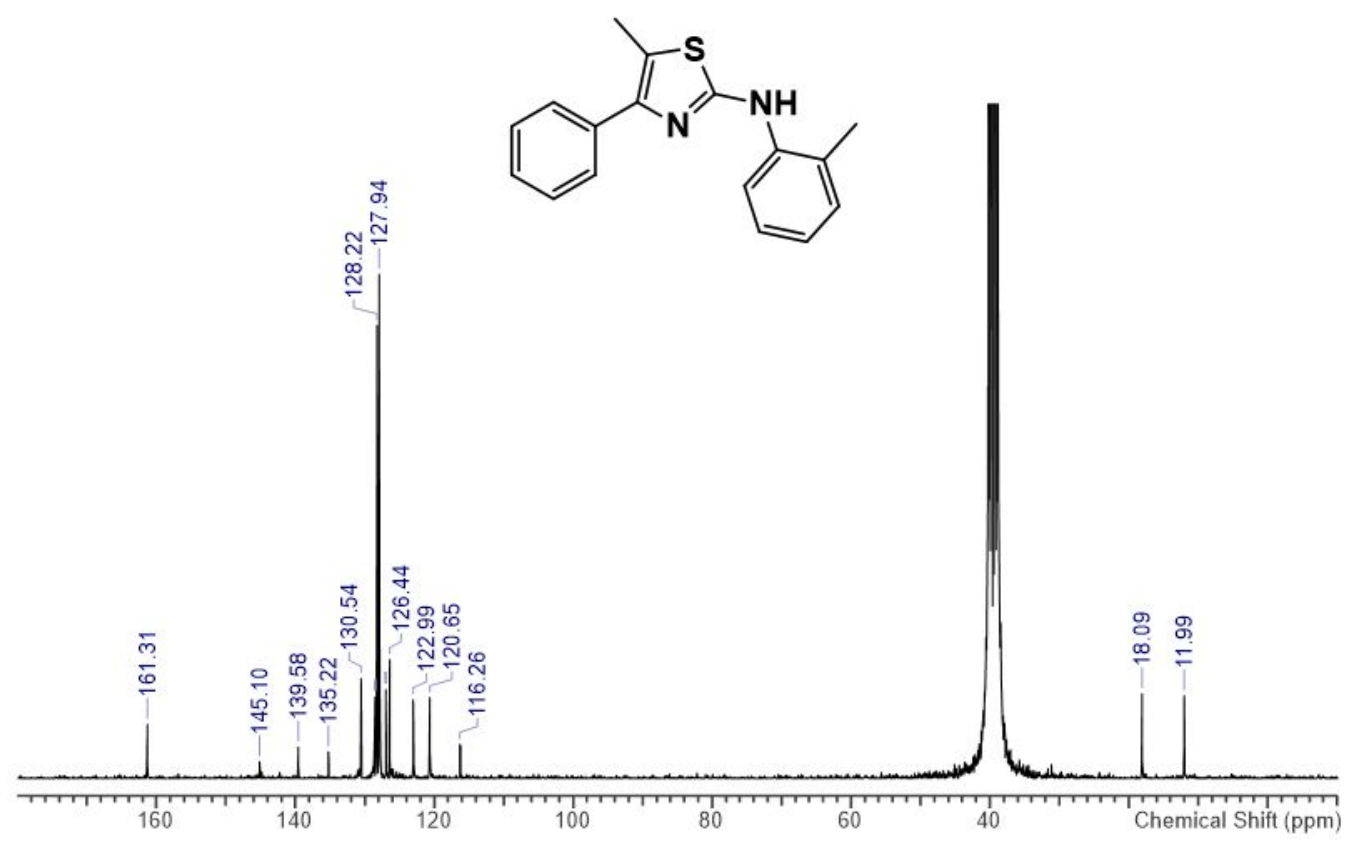

Figure S24. ${ }^{13} \mathrm{C}\{\mathrm{H}\}-\mathrm{NMR}$ spectrum of thiazole $9 \mathrm{~b}\left(\mathrm{DMSO}-\mathrm{d}_{6}, 100 \mathrm{MHz}, 25{ }^{\circ} \mathrm{C}\right)$. 


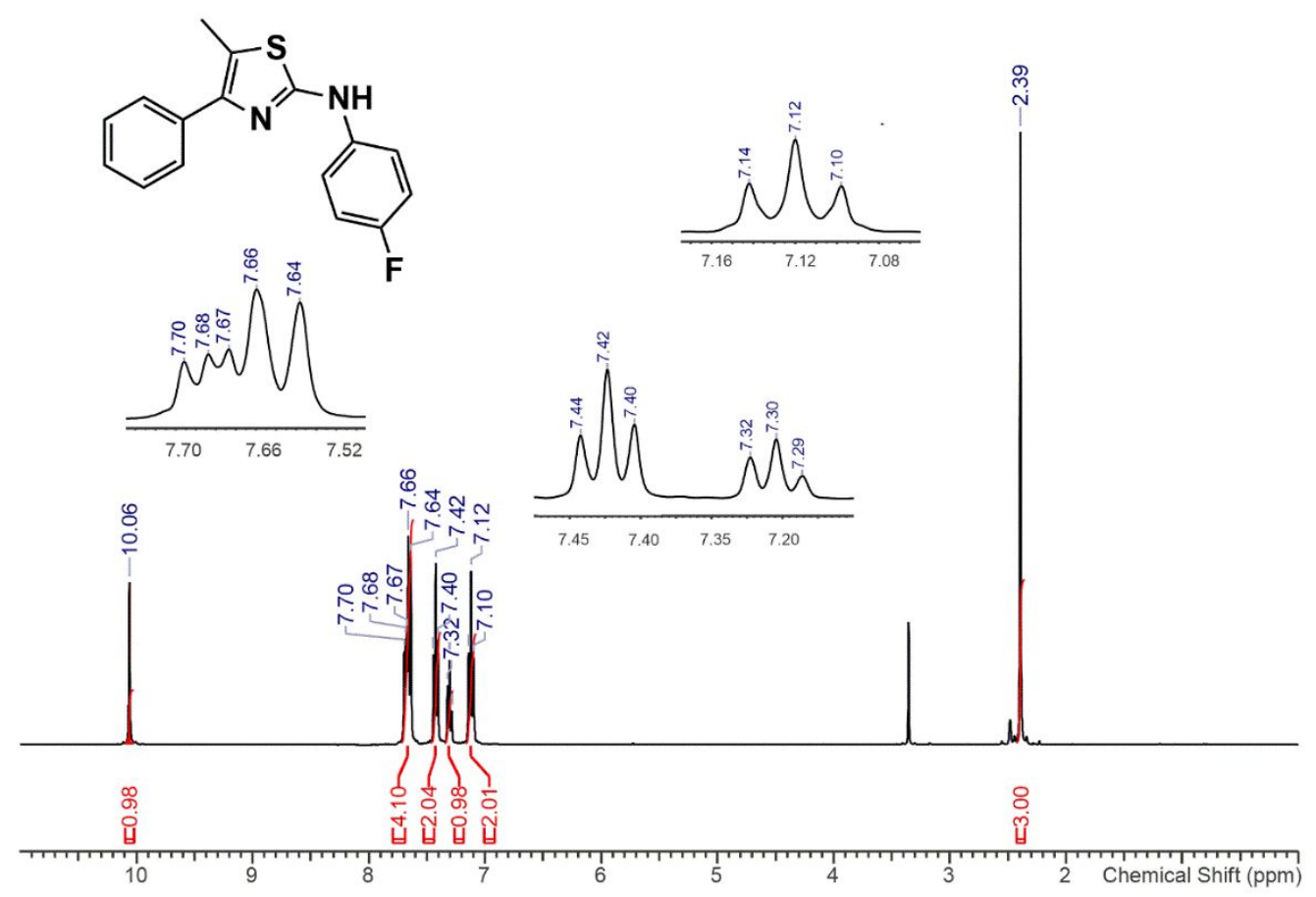

Figure S25. ${ }^{1} \mathrm{H}-\mathrm{NMR}$ spectrum of thiazole $14 \mathbf{b}\left(\mathrm{DMSO}_{-} \mathrm{d}_{6}, 400 \mathrm{MHz}, 25{ }^{0} \mathrm{C}\right)$.

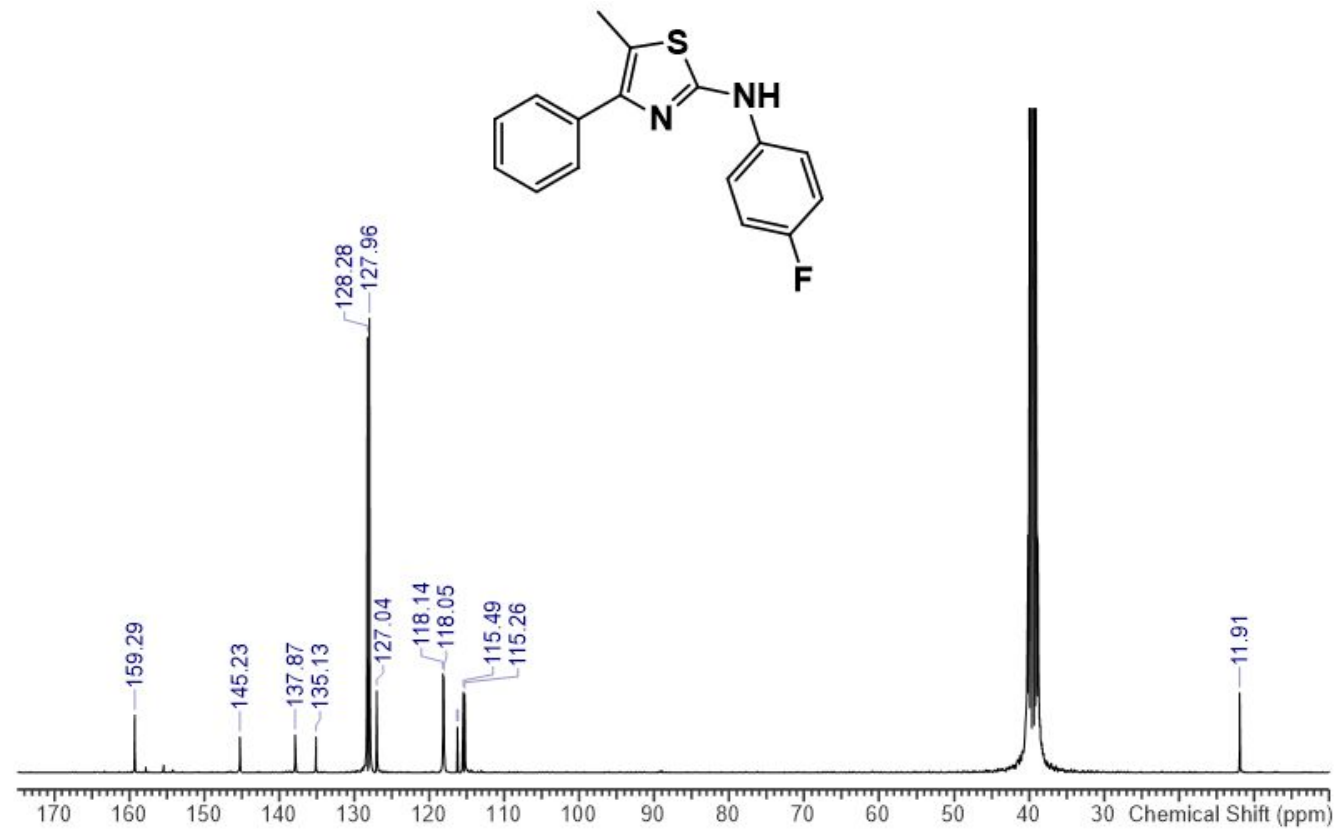

Figure S26. ${ }^{13} \mathrm{C}\{\mathrm{H}\}-\mathrm{NMR}$ spectrum of thiazole 14b (DMSO-d $\left.6,100 \mathrm{MHz}, 25{ }^{0} \mathrm{C}\right)$. 


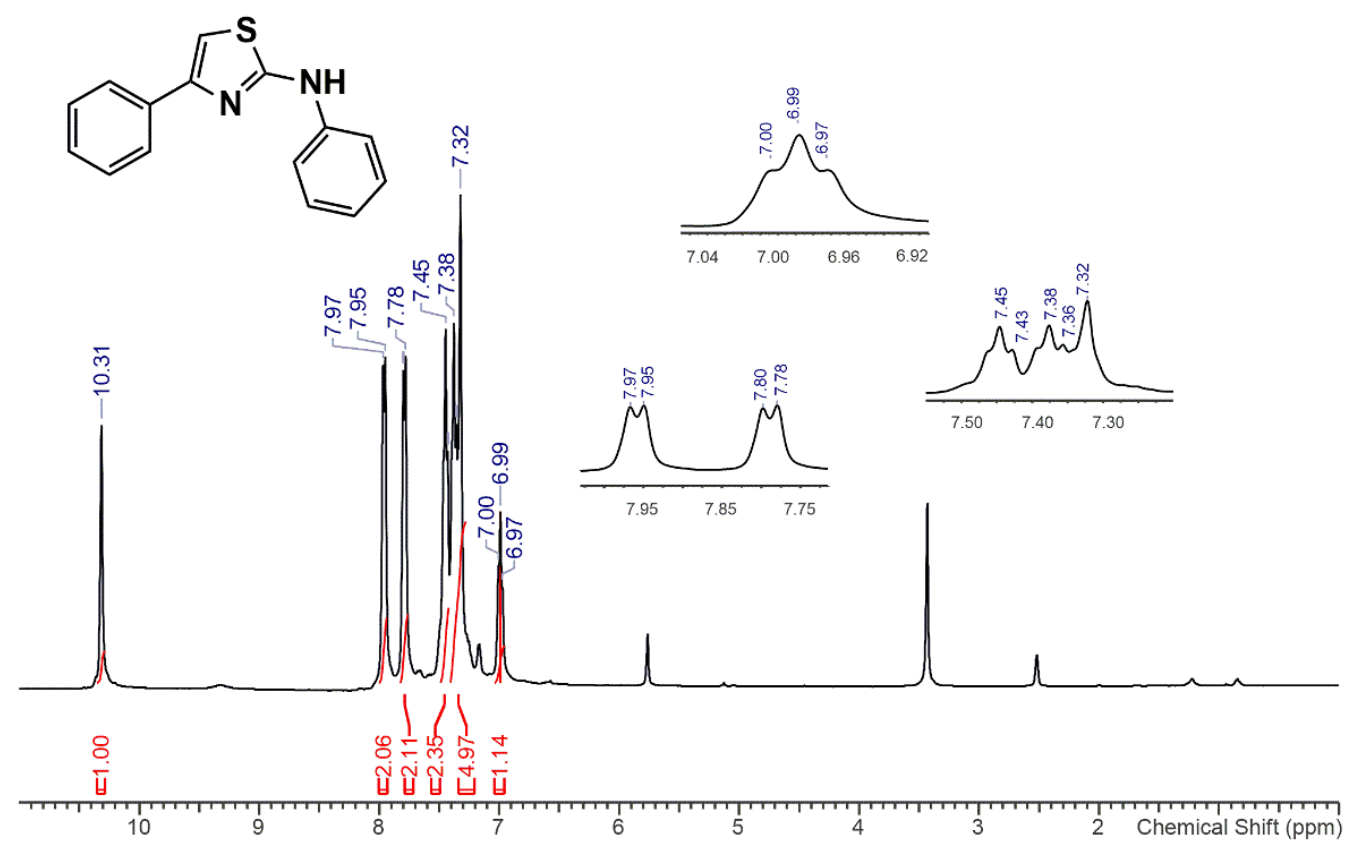

Figure S27. ${ }^{1} \mathrm{H}-\mathrm{NMR}$ spectrum of thiazole $17 \mathbf{b}\left(\mathrm{DMSO}^{-} \mathrm{d}_{6}, 400 \mathrm{MHz}, 25^{0} \mathrm{C}\right)$.

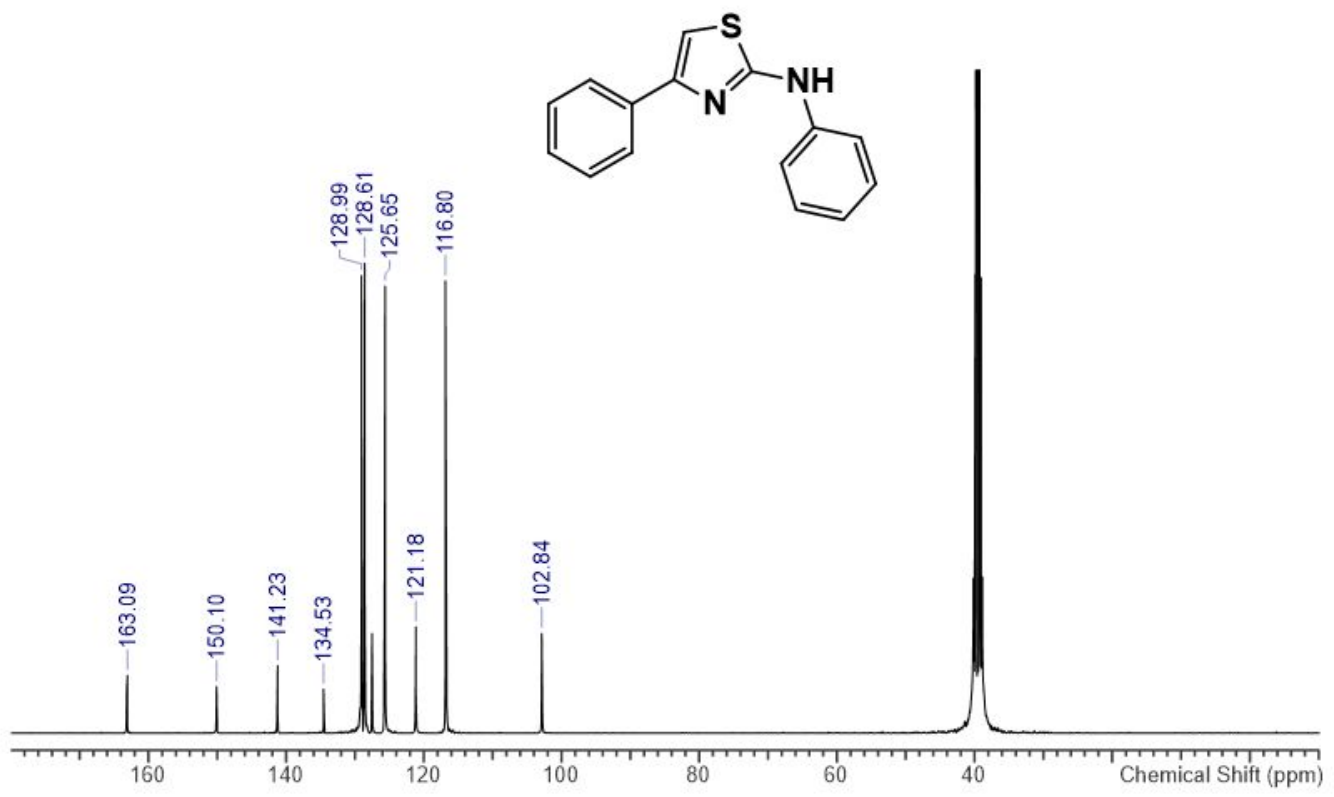

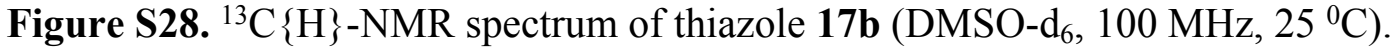




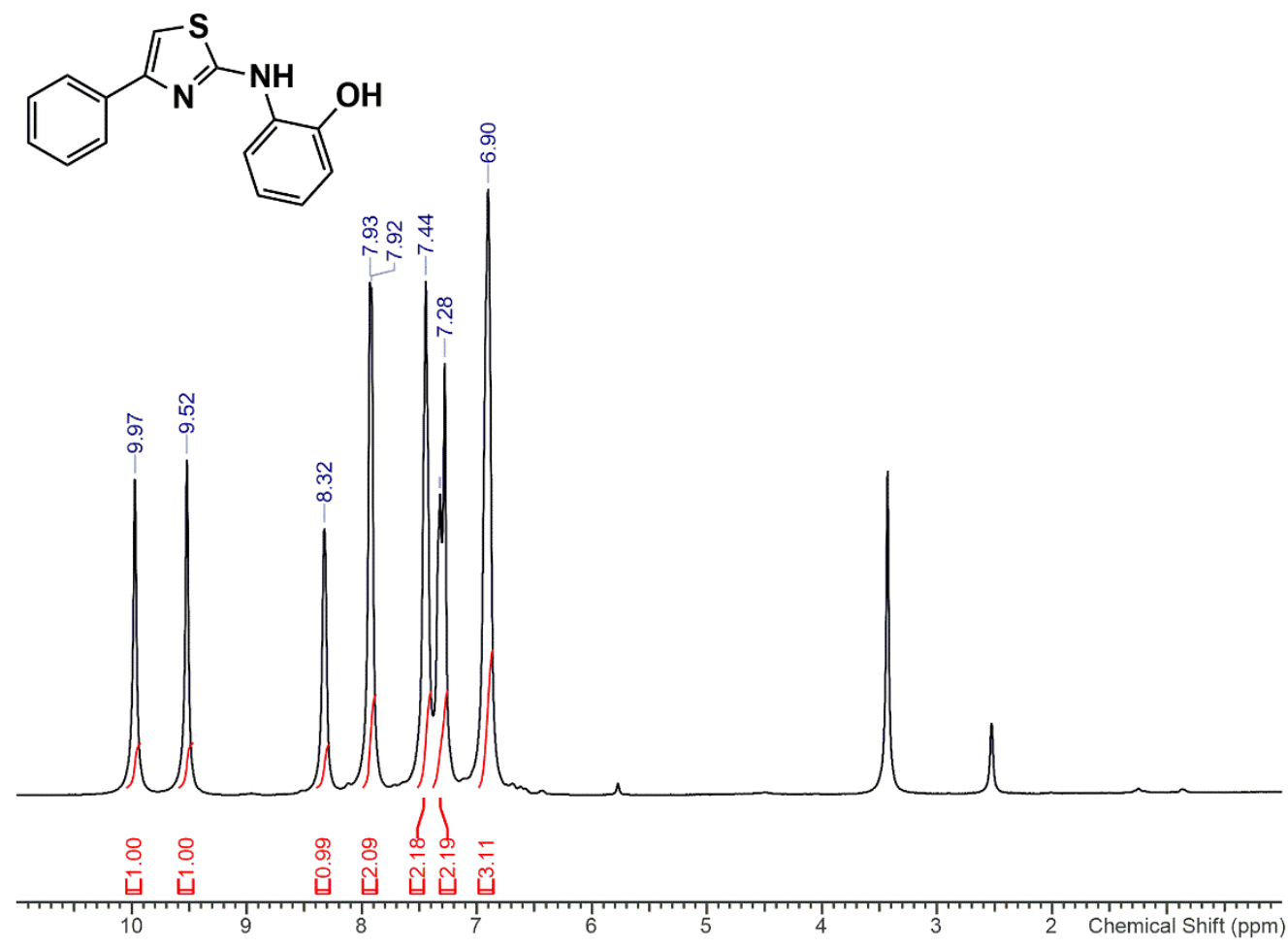

Figure S29. ${ }^{1} \mathrm{H}-\mathrm{NMR}$ spectrum of thiazole $18 \mathbf{b}\left(\mathrm{DMSO}_{-} \mathrm{d}_{6}, 400 \mathrm{MHz}, 25{ }^{0} \mathrm{C}\right)$.

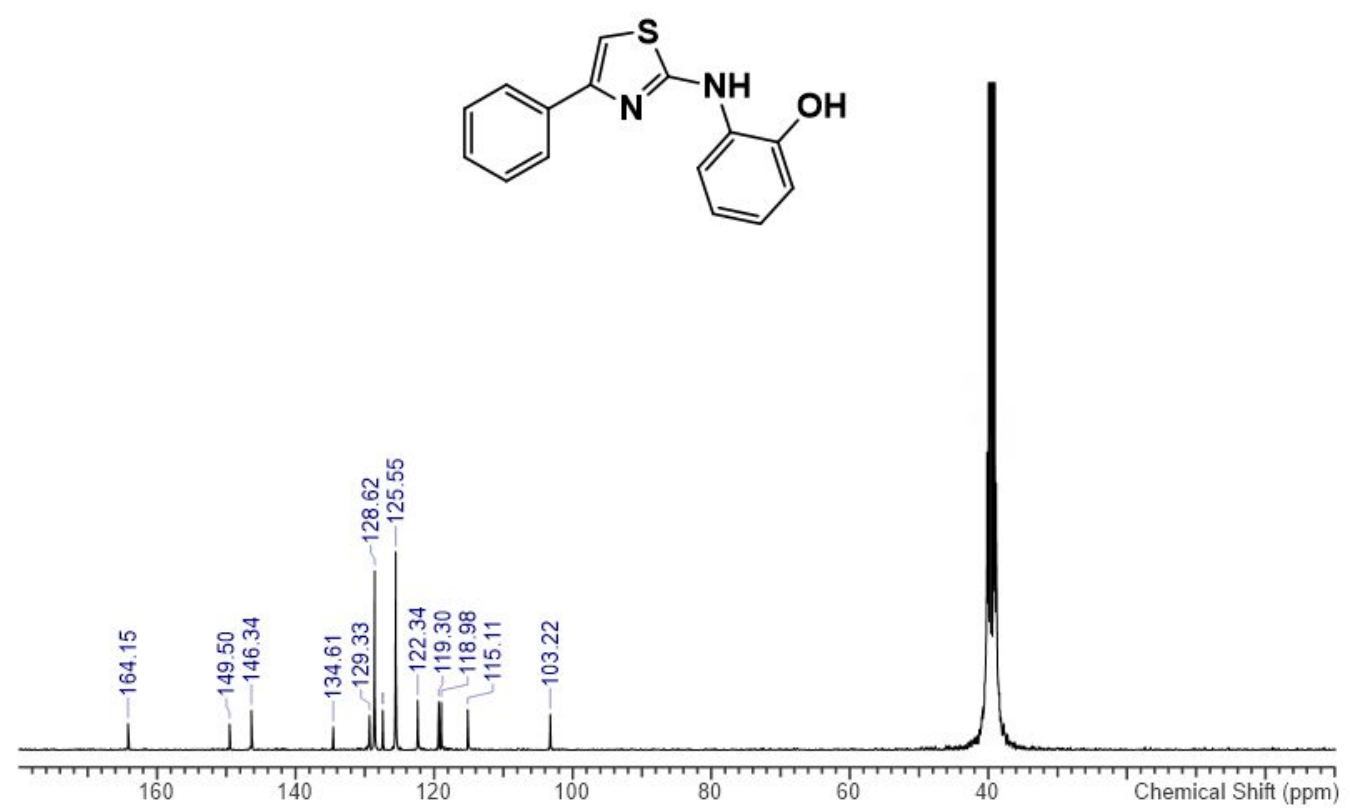

Figure S30. ${ }^{13} \mathrm{C}\{\mathrm{H}\}-\mathrm{NMR}$ spectrum of thiazole $\mathbf{1 8 b}\left(\mathrm{DMSO}_{6}, 100 \mathrm{MHz}, 25{ }^{0} \mathrm{C}\right)$. 


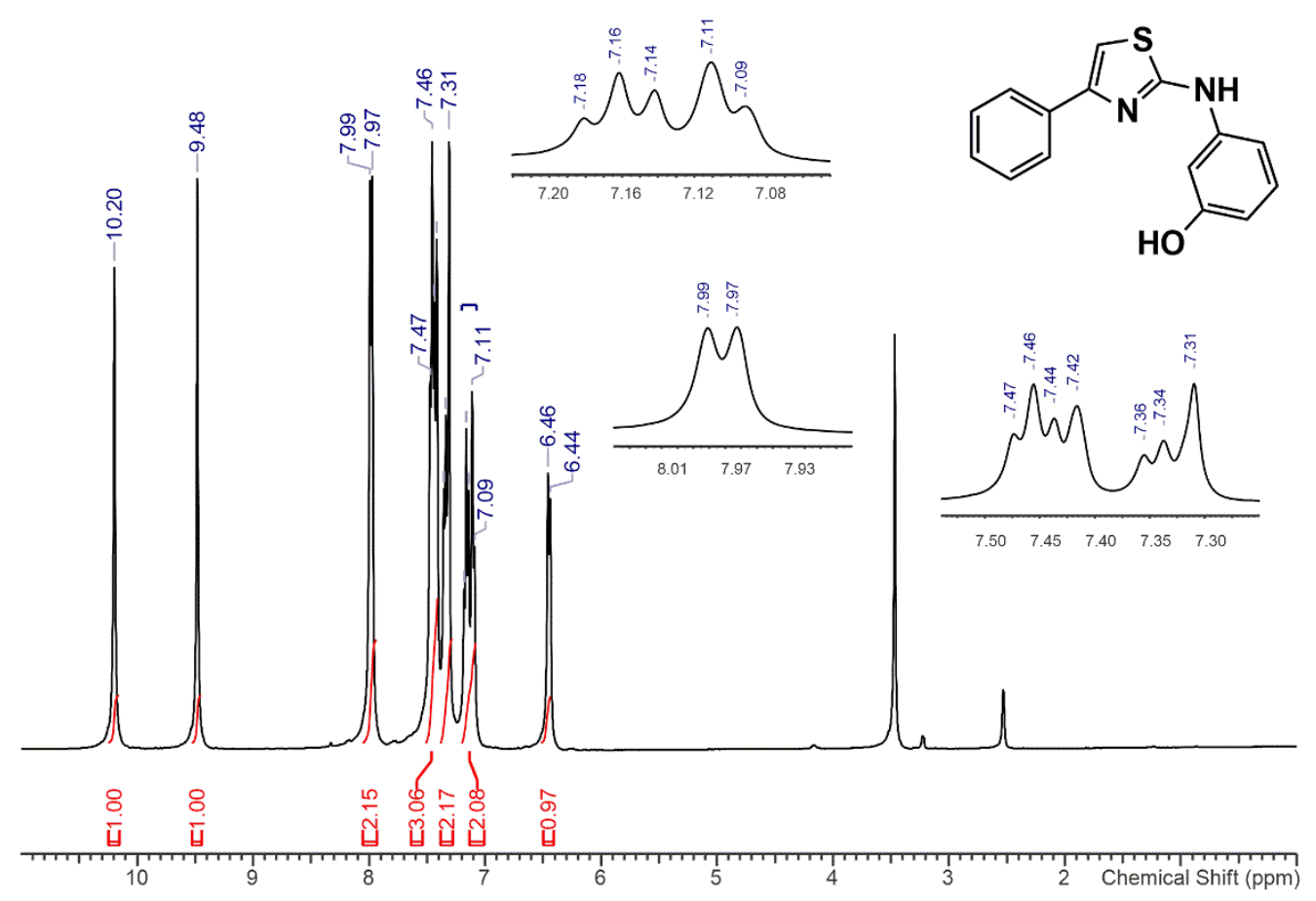

Figure S31. ${ }^{1} \mathrm{H}-\mathrm{NMR}$ spectrum of thiazole 19b (DMSO- $\mathrm{d}_{6}, 400 \mathrm{MHz}, 2{ }^{0} \mathrm{C}$ ).

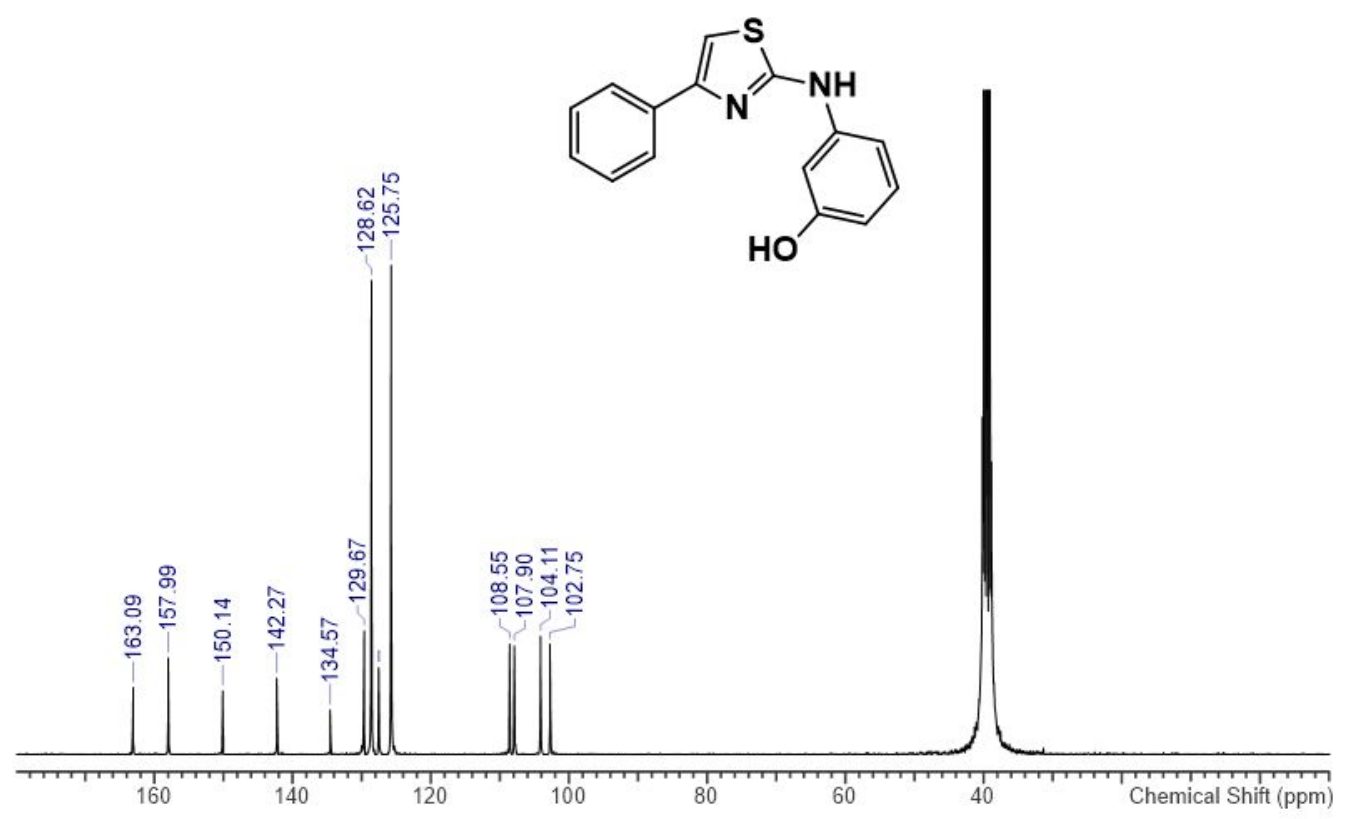

Figure S32. ${ }^{13} \mathrm{C}\{\mathrm{H}\}-\mathrm{NMR}$ spectrum of thiazole $19 \mathrm{~b}\left(\mathrm{DMSO}-\mathrm{d}_{6}, 100 \mathrm{MHz}, 25{ }^{0} \mathrm{C}\right)$. 


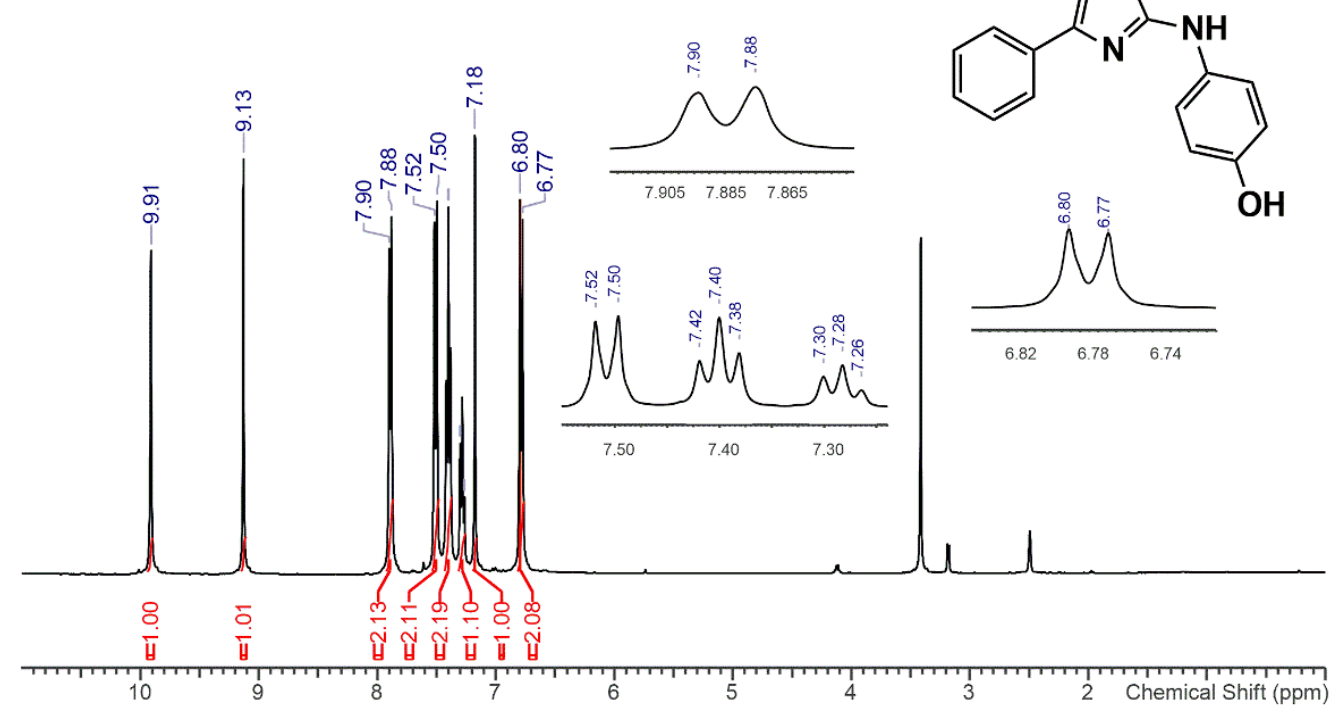

Figure S33. ${ }^{1} \mathrm{H}-\mathrm{NMR}$ spectrum of thiazole $20 \mathrm{~b}\left(\mathrm{DMSO}-\mathrm{d}_{6}, 400 \mathrm{MHz}, 25^{0} \mathrm{C}\right)$.

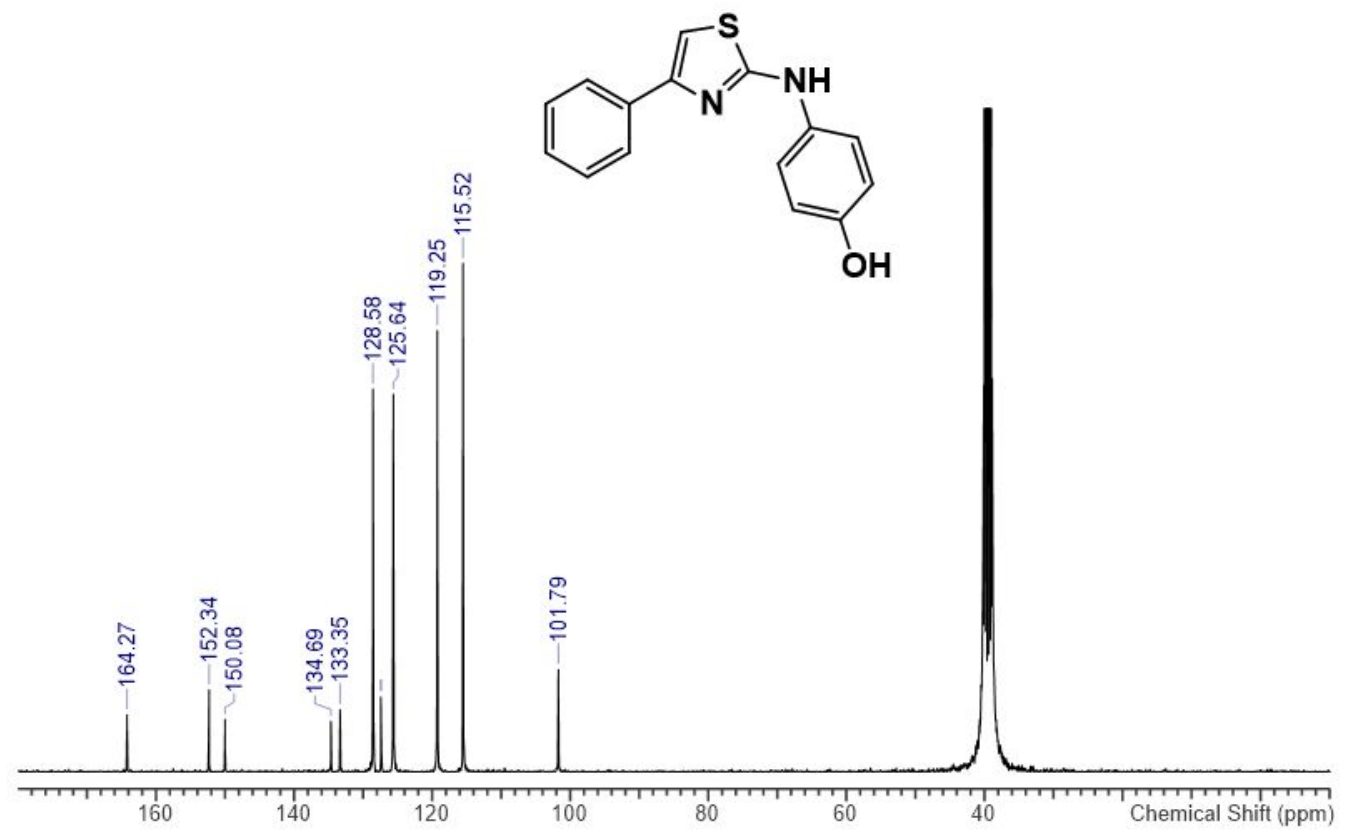

Figure S34. ${ }^{13} \mathrm{C}\{\mathrm{H}\}-\mathrm{NMR}$ spectrum of thiazole $20 \mathrm{~b}\left(\mathrm{DMSO}-\mathrm{d}_{6}, 100 \mathrm{MHz}, 25{ }^{0} \mathrm{C}\right)$. 
<smiles>Cc1ccccc1Nc1nc(-c2ccccc2)cs1</smiles>
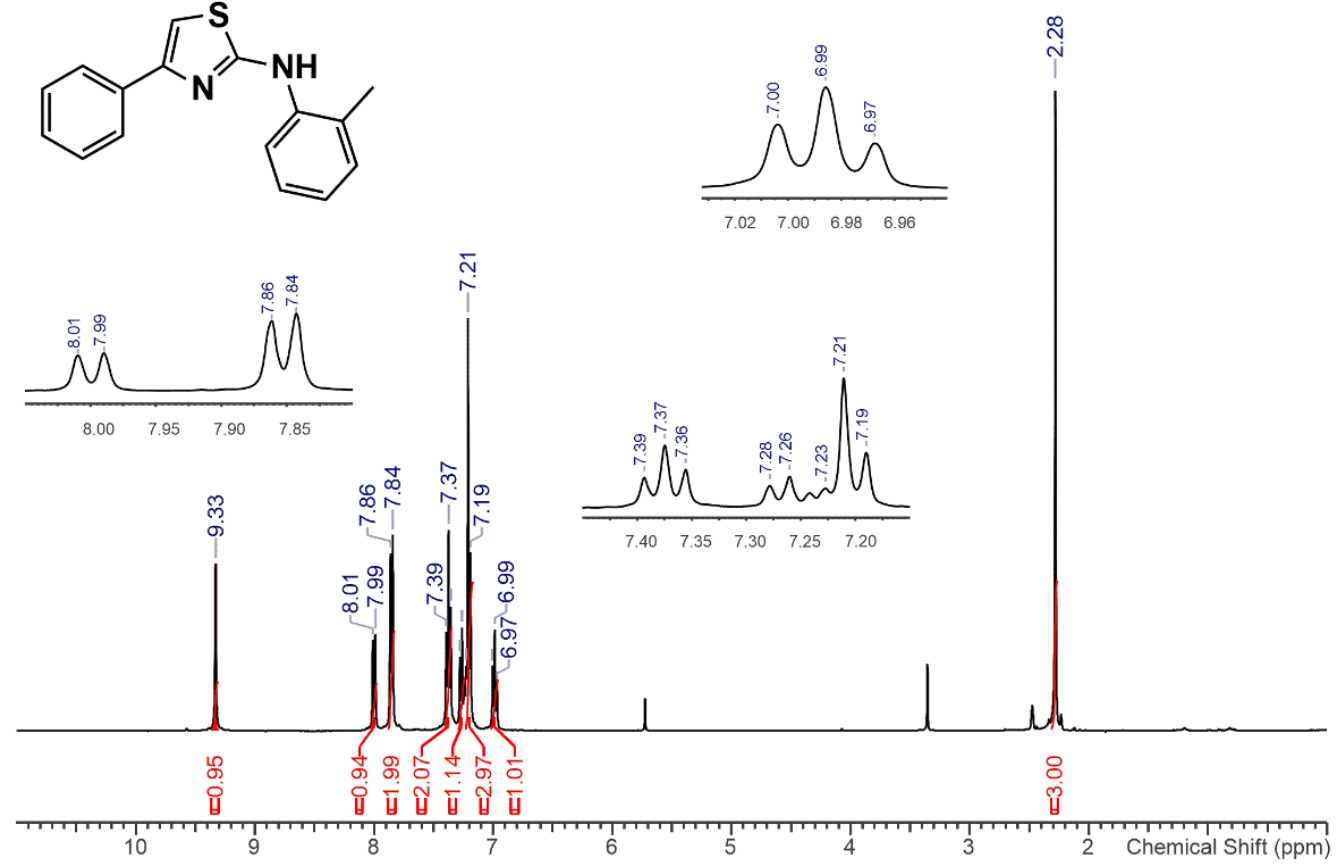

Figure S35. ${ }^{1} \mathrm{H}-\mathrm{NMR}$ spectrum of thiazole $21 \mathrm{~b}\left(\mathrm{DMSO}-\mathrm{d}_{6}, 400 \mathrm{MHz}, 25^{\circ} \mathrm{C}\right)$.

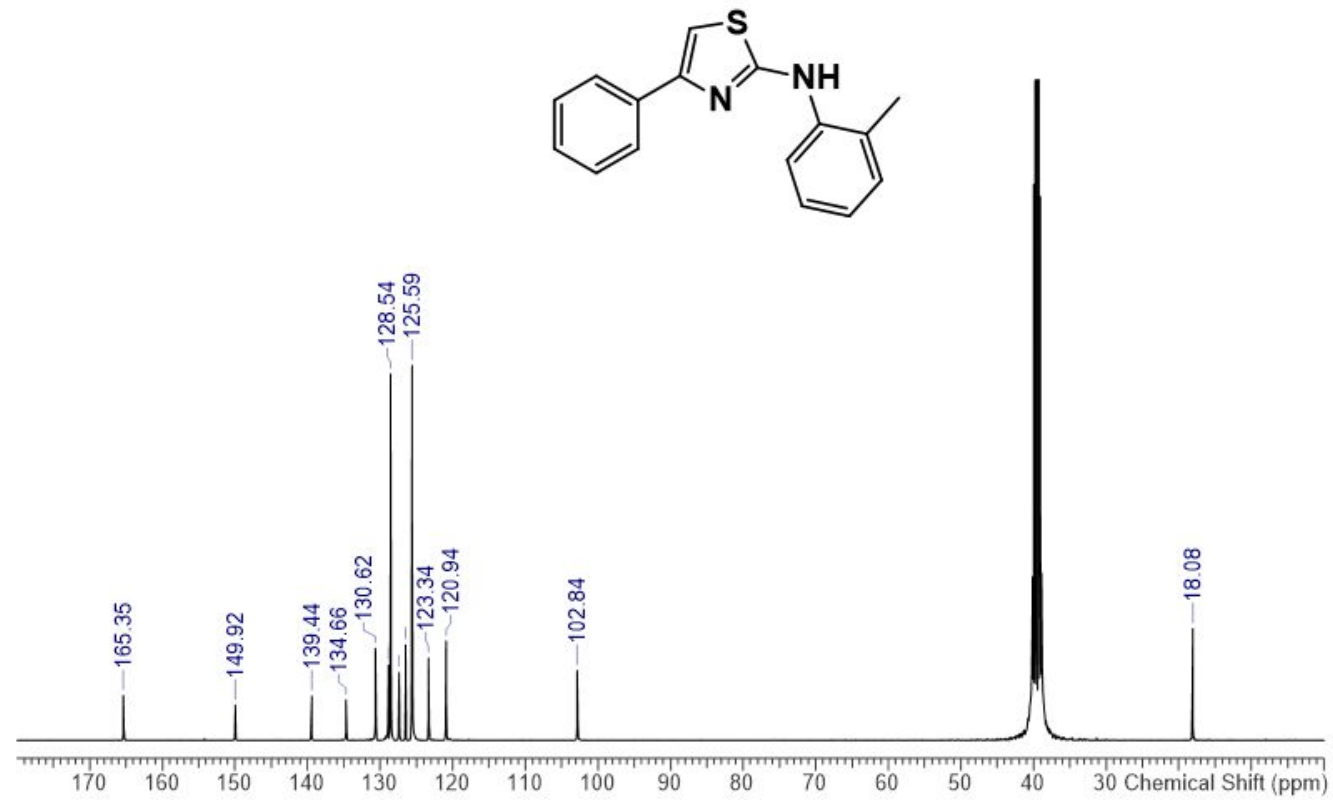

Figure S36. ${ }^{13} \mathrm{C}\{\mathrm{H}\}-\mathrm{NMR}$ spectrum of thiazole $21 \mathbf{b}\left(\mathrm{DMSO}_{-} \mathrm{d}_{6}, 100 \mathrm{MHz}, 25{ }^{0} \mathrm{C}\right)$. 
<smiles>Cc1ccc(Nc2nc(-c3ccccc3)cs2)cc1</smiles>
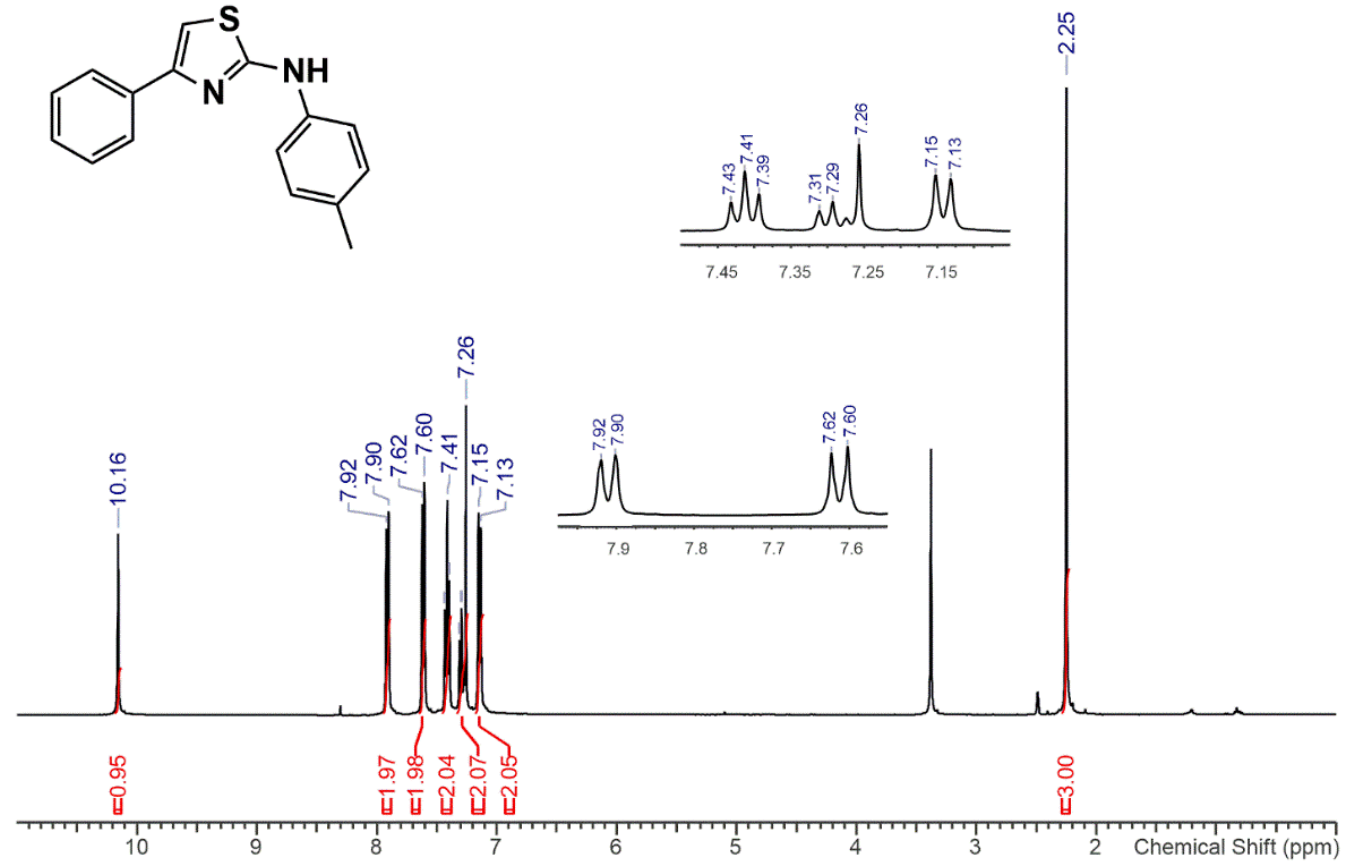

Figure S37. ${ }^{1} \mathrm{H}-\mathrm{NMR}$ spectrum of thiazole 22b $\left(\mathrm{DMSO}^{-} \mathrm{d}_{6}, 400 \mathrm{MHz}, 25^{\circ} \mathrm{C}\right)$.

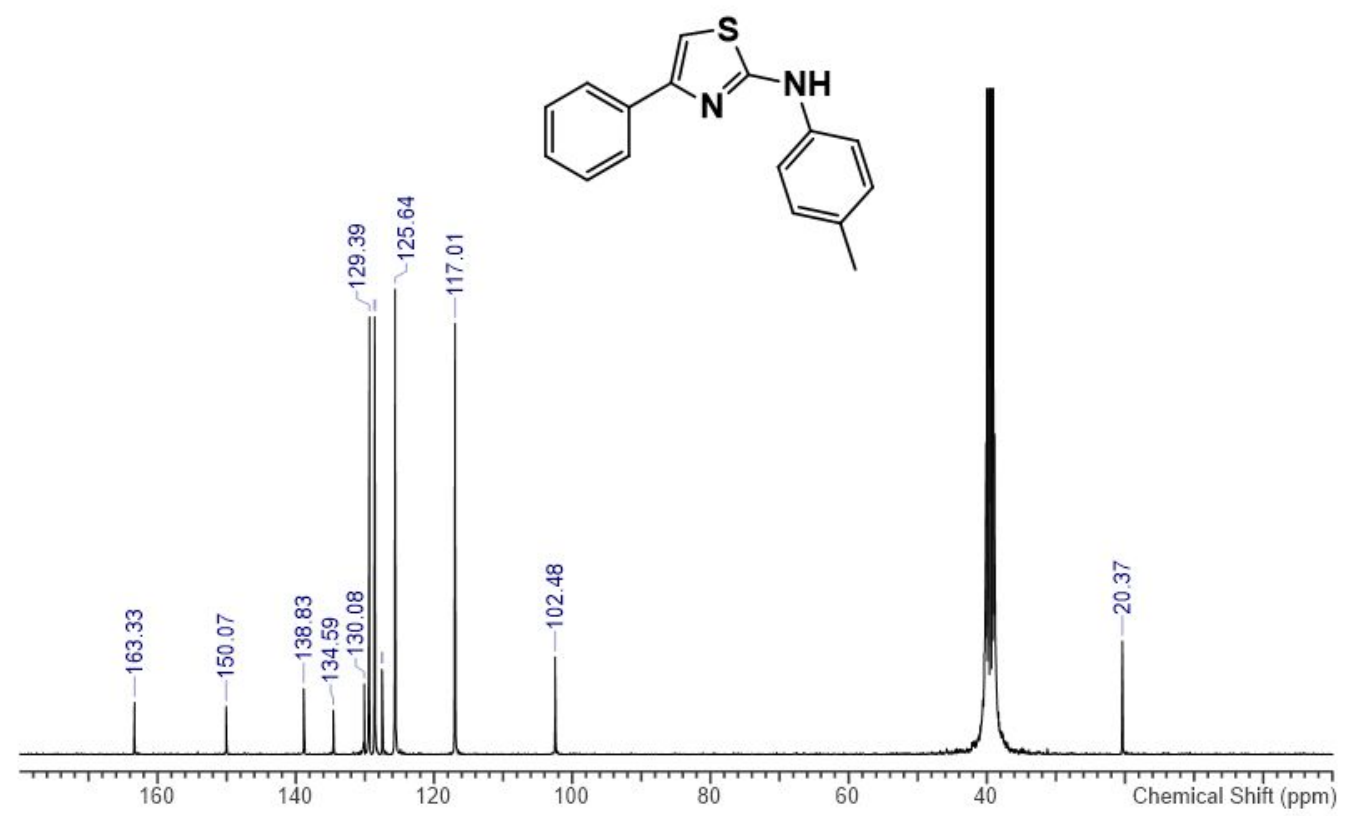

Figure S38. ${ }^{13} \mathrm{C}\{\mathrm{H}\}-\mathrm{NMR}$ spectrum of thiazole $22 \mathbf{b}\left(\mathrm{DMSO}-\mathrm{d}_{6}, 100 \mathrm{MHz}, 25{ }^{0} \mathrm{C}\right)$. 


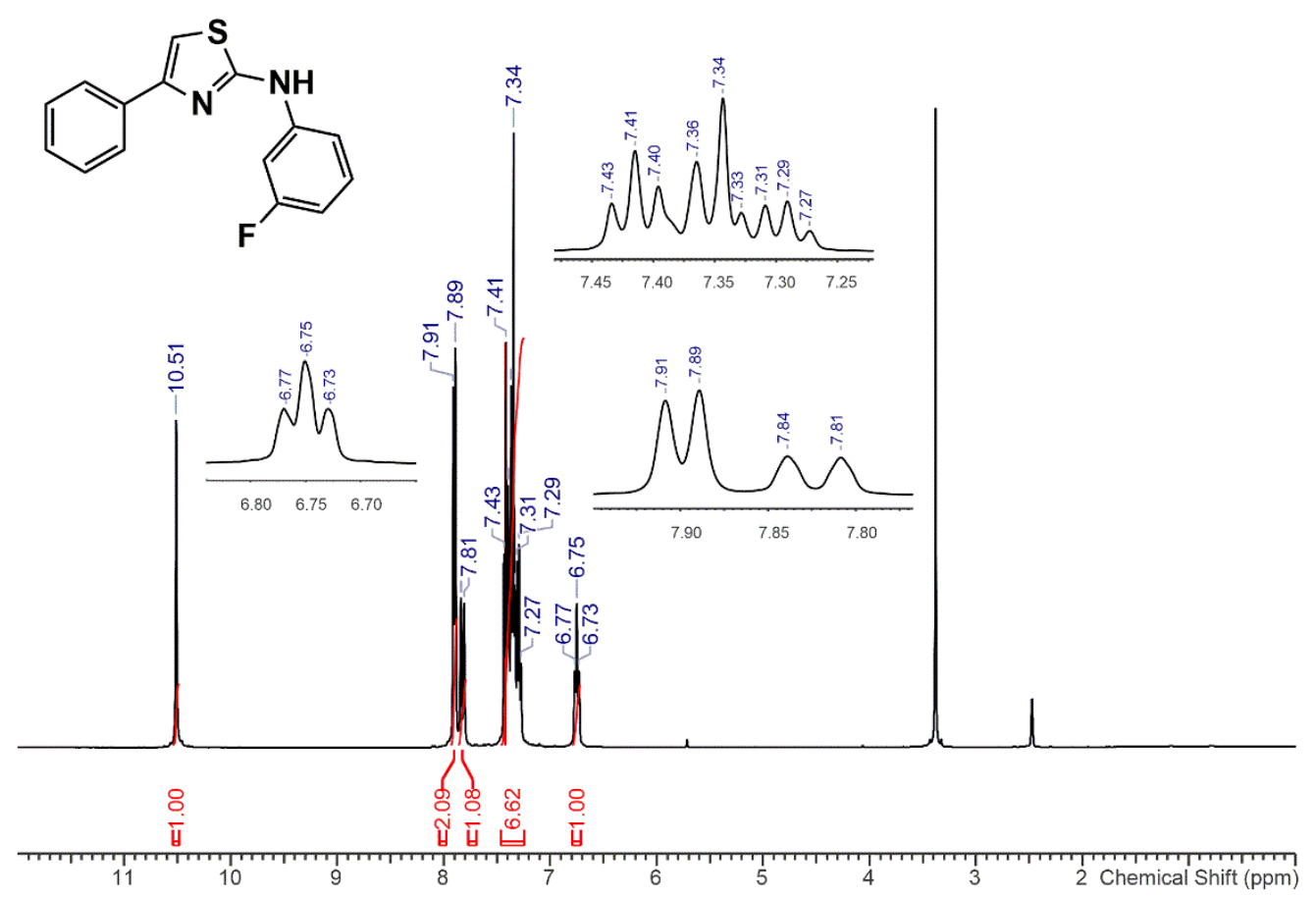

Figure S39. ${ }^{1} \mathrm{H}-\mathrm{NMR}$ spectrum of thiazole 23b (DMSO- $\left.\mathrm{d}_{6}, 400 \mathrm{MHz}, 25{ }^{0} \mathrm{C}\right)$.<smiles>Fc1cccc(Nc2nc(-c3ccccc3)cs2)c1</smiles>

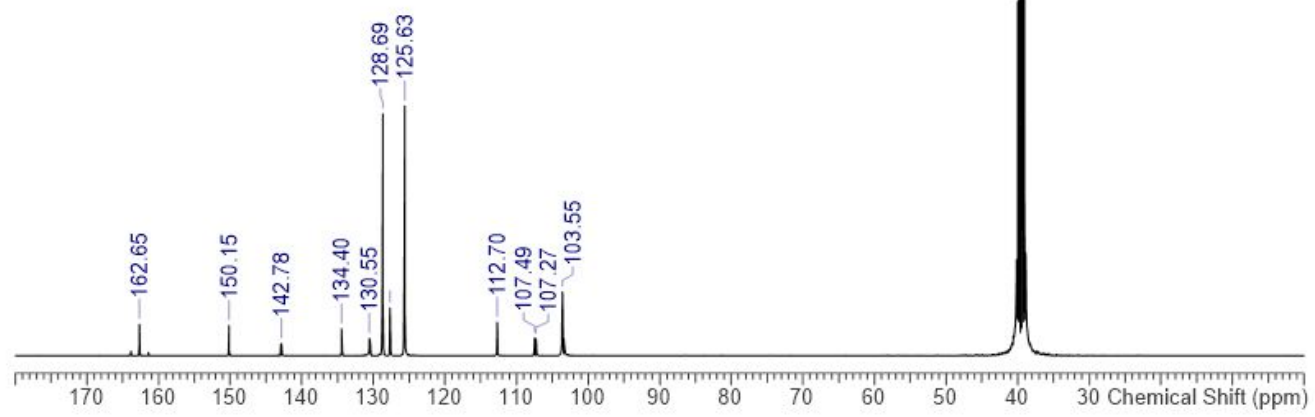

Figure S40. ${ }^{13} \mathrm{C}\{\mathrm{H}\}-\mathrm{NMR}$ spectrum of thiazole $\mathbf{2 3 b}\left(\mathrm{DMSO}_{-} \mathrm{d}_{6}, 100 \mathrm{MHz}, 25{ }^{0} \mathrm{C}\right)$. 


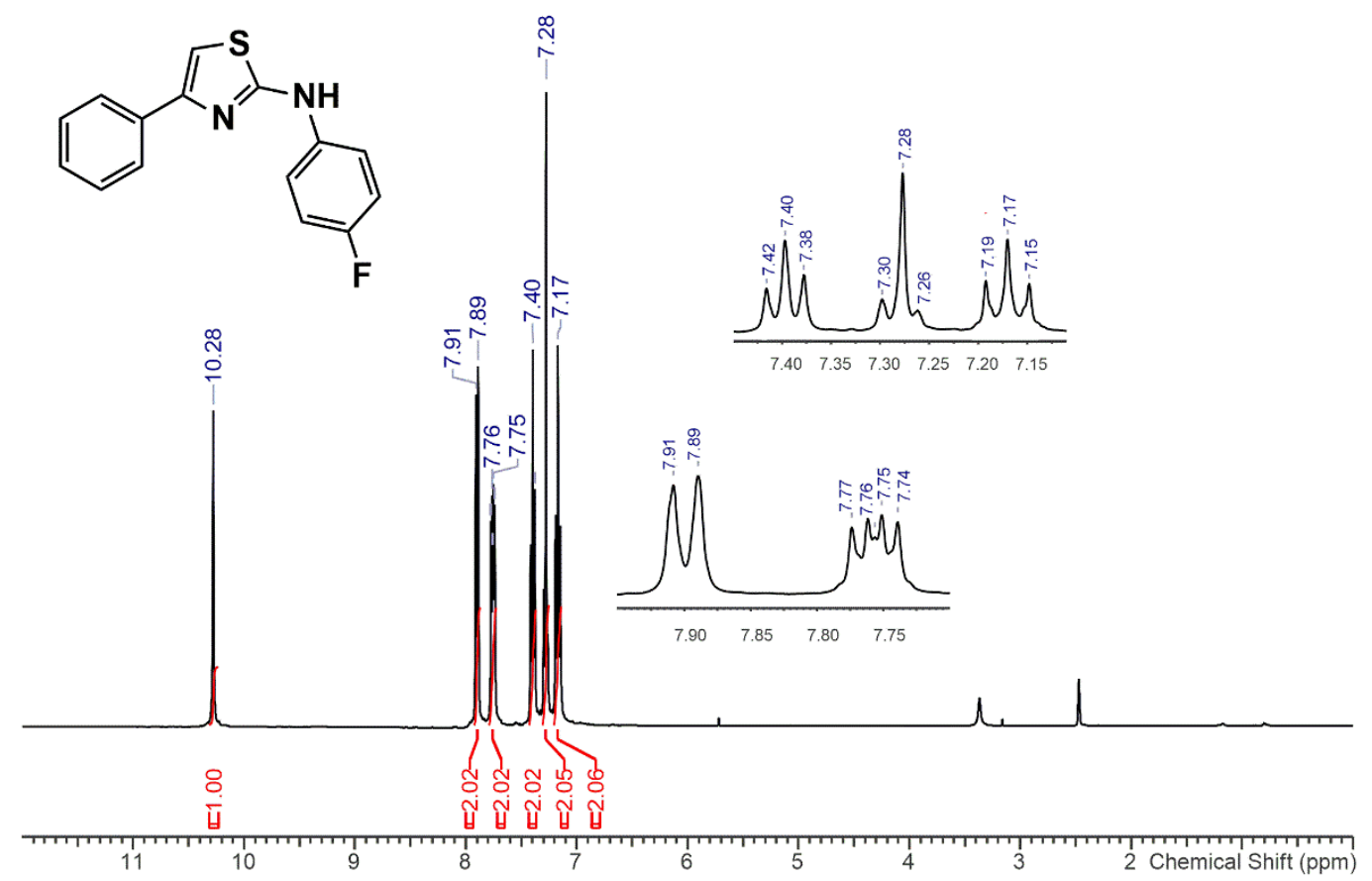

Figure S41. ${ }^{1} \mathrm{H}-\mathrm{NMR}$ spectrum of thiazole $24 \mathbf{b}\left(\mathrm{DMSO}_{-} \mathrm{d}_{6}, 400 \mathrm{MHz}, 2{ }^{0} \mathrm{C}\right)$.<smiles>Fc1ccc(Nc2nc(-c3ccccc3)cs2)cc1</smiles>

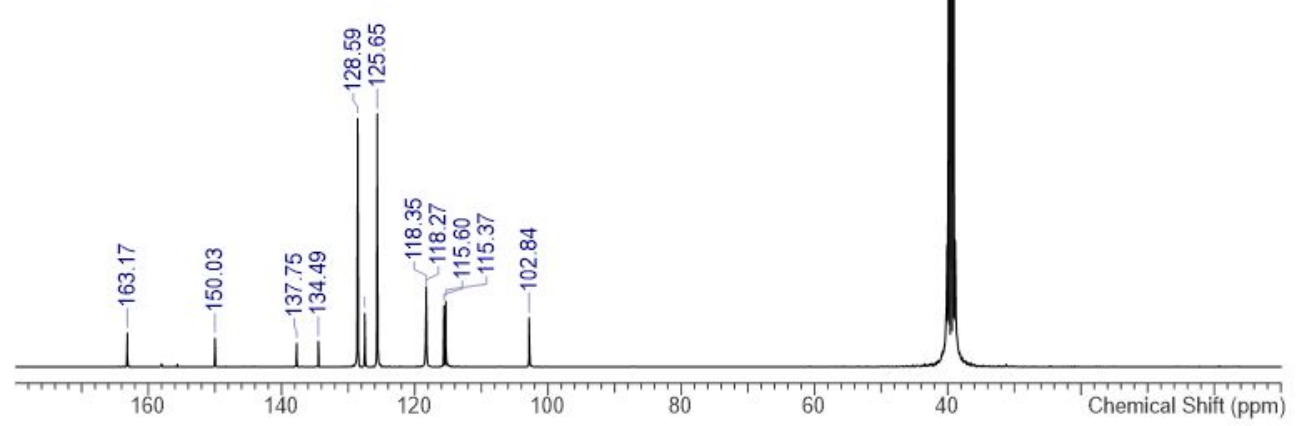

Figure S42. ${ }^{13} \mathrm{C}\{\mathrm{H}\}-\mathrm{NMR}$ spectrum of thiazole $24 \mathbf{b}\left(\mathrm{DMSO}_{-} \mathrm{d}_{6}, 100 \mathrm{MHz}, 25{ }^{0} \mathrm{C}\right)$. 


\section{NMR Spectra}

\section{HRMS (ESI) data for thiazole $2 b$}

The mass spectral analyses were performed on an Aligent Technologies 6540 UHD AccurateMass Q-TOF LC/MS.

HRMS (ESI) m/z: $(\mathrm{M}+\mathrm{H})^{+}$Calcd for $\mathrm{C}_{16} \mathrm{H}_{15} \mathrm{~N}_{2} \mathrm{OS}^{+}$283.0900; Found 283.0907

MS Spectrum Peak List
\begin{tabular}{|l|l|l|l|l|l|}
\hline $\boldsymbol{m} / \boldsymbol{z}$ & $\mathrm{z}$ & \multicolumn{1}{l}{ Abund } & Formula & Ion \\
\hline 283.0907 & 1 & 297949.94 & $\mathrm{C} 16 \mathrm{H} 14 \mathrm{~N} 2 \mathrm{OS}$ & $(\mathrm{M}+\mathrm{H})+$ \\
\hline 284.0934 & 1 & 52030.02 & $\mathrm{C} 16 \mathrm{H} 14 \mathrm{~N} 2 \mathrm{OS}$ & $(\mathrm{M}+\mathrm{H})+$ \\
\hline 285.0892 & 1 & 15238.81 & $\mathrm{C} 16 \mathrm{H} 14 \mathrm{~N} 2 \mathrm{OS}$ & $(\mathrm{M}+\mathrm{H})+$ \\
\hline 305.0733 & 1 & 591.61 & $\mathrm{C} 16 \mathrm{H} 14 \mathrm{~N} 2 \mathrm{OS}$ & $(\mathrm{M}+\mathrm{Na})+$ \\
\hline 306.0852 & 1 & 100.5 & $\mathrm{C} 16 \mathrm{H} 14 \mathrm{~N} 2 \mathrm{OS}$ & $(\mathrm{M}+\mathrm{Na})+$ \\
\hline & 565.2594 & 1 & 206.15 & $\mathrm{C} 16 \mathrm{H} 14 \mathrm{~N} 2 \mathrm{OS}$ & $(2 \mathrm{M}+\mathrm{H})+$ \\
\hline & 587.2515 & 1 & 255.74 & $\mathrm{C} 16 \mathrm{H} 14 \mathrm{~N} 2 \mathrm{OS}$ & $(2 \mathrm{M}+\mathrm{Na})+$ \\
\hline & 588.2476 & 1 & 140.94 & $\mathrm{C} 16 \mathrm{H} 14 \mathrm{~N} 2 \mathrm{OS}$ & $(2 \mathrm{M}+\mathrm{Na})+$ \\
\hline
\end{tabular}

MS Spectrum

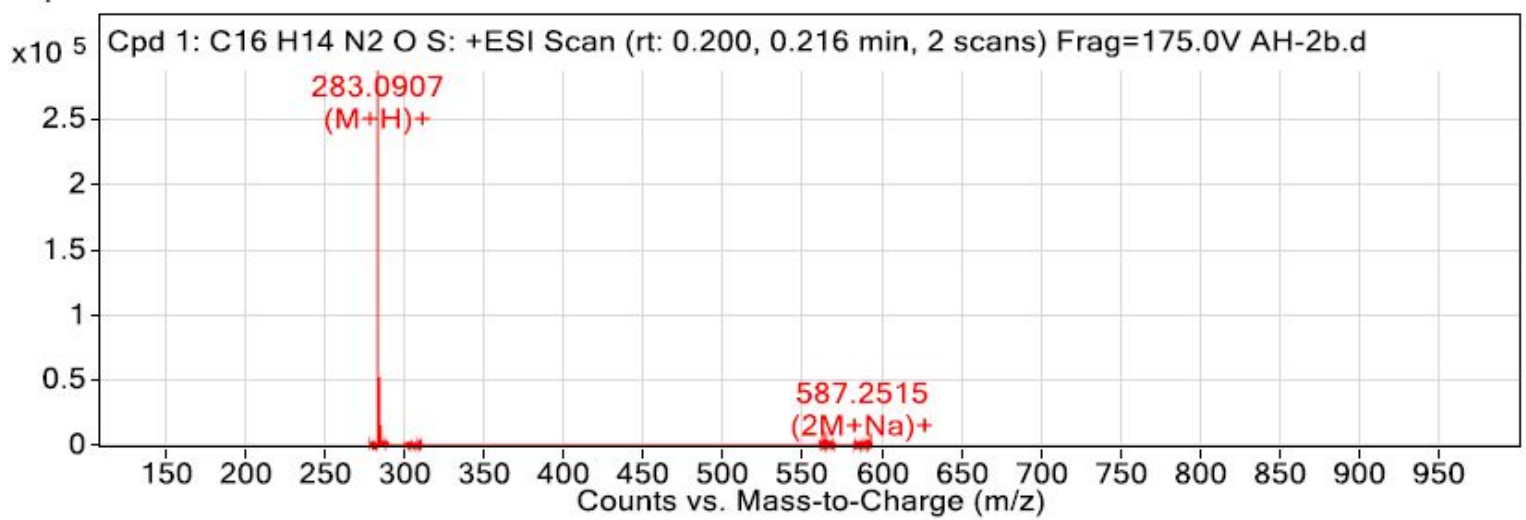

MS Zoomed Spectrum

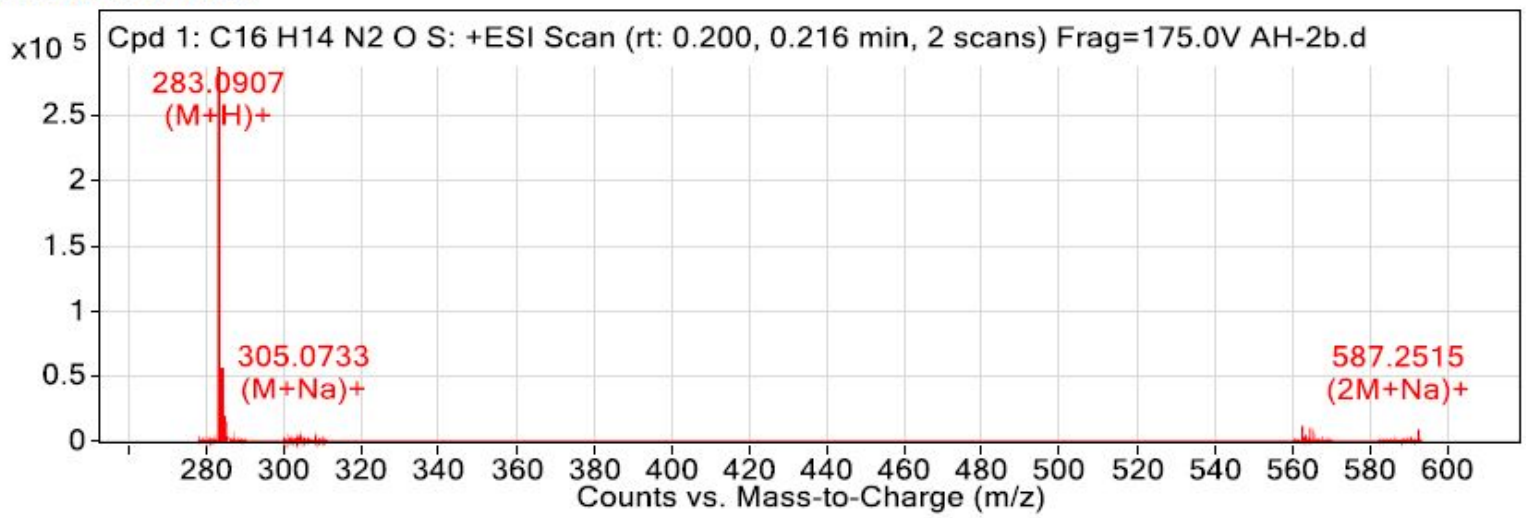

Figure S43. ESI-HRMS spectral data for thiazole $\mathbf{2 b}$. 